\title{
Centromeric repeats of the Western European house mouse II: Selection for high local diversity of monomers and a hypothesis for coevolution between centromere size and karyotype number
}

\author{
William R. Rice* \\ *Department of Ecology, Evolution and Marine Biology, University of California, Santa Barbara, CA 93106, USA, \\ email: rice@lifesci.ucsb.edu
}

Key Words: Centromere, karyotype evolution, centromere drive, Mus musculus domesticus

The companion paper (Rice 2020) found that the centromeric repeats of the Western European house mouse (Mus musculus domesticus) have unusual structure: i) despite moderate pairwise sequence divergence (average $=5.9 \%$ ), no monomer sequence was common and many hundreds of monomer sequences were observed, ii) local sequence divergence among neighboring monomers was nearly as high as genome-wide divergence, and iii) matching sequences were rare between side-by-side monomers. Here I integrate information from many published studies to formulate a hypothesis for the evolution of this structure. Non-matching sequences of neighboring centromeric monomers is hypothesized to be selectively favored in the context of molecular drive because it reduces the rate of monomer deletion during repair of double strand breaks (DSBs) via the Single Strand Annealing (SSA) pathway. The foundation for the hypothesis is the observation that centromeres of most populations of $M$. $\mathbf{m}$. domestics reside close to the telomere, i.e., all their chromosomes are telocentrics. This proximity influences repair of centromeric DSBs because it places at least part of the centromere within the Telomere-Affected Repair Region (TARR; a location with increased concentrations of the shelterin-complex proteins that bind telomeres, especially TRF2). Shelterin proteins increase the level of 5' $\rightarrow 3$ ' end resection at DSBs and thereby: i) decrease the frequency of repair via the c-NHEJ pathway, and ii) increase the frequency of homology-directed repair (HD-repair) -including the SSA repair pathway. It is hypothesized that certain 'trigger' events (e.g., sub-telomeric deletions) occur in local populations that increase the influence of TARR on the centromere. This increase elevates the occurrence of SSA repair of centromeric DSBs to a level that causes centromeres to begin to gradually shrink. Chronic shrinkage leads to coevolution between centromere size and karyotype number. Once centromeres shrink to a size below a critical minimum (that causes substantially reduced kinetochore size), fusions between non-homologous telocentrics with undersized centromeres produces metacentrics with an expanded centromere size (and a corresponding 'quantum-jump' in kinetochore size). These metacentrics: i) accumulate to fixation because they are favored by centromere drive, and ii) are released from the influence of TARR and thereby gradually recover larger centromere size. Fission of metacentrics with enlarged centromeres can next plausibly regenerate pairs of telocentrics with sufficiently large centromeres (which recruit normal-sized kinetochores) to be favored by centromere drive and accumulate to fixation. This fixation completes a cycle of coevolution within genomes that oscillate between two extremes: i) high karyotype number $(2 \mathrm{~N}=40$; all telocentrics) with larger centromeres, and ii) low karyotype number $(2 \mathrm{~N} \ll 40$; mainly metacentrics) with initially small centromeres that gradually increase in size.

\section{Introduction}

The Western European house mouse (Mus musculus domesticus) is unusual because of its extreme variation in karyotype among different local populations. Their diploid numbers (2N) span a range from 22 (nearly all metacentrics) to 40 (all telocentrics) $X$ and autosomes (reviewed in Nachman and Searle 1995; Garagna et al. 2014). This karyotypic variation is highly uneven: a large majority of populations have $2 \mathrm{~N}=40$ telocentrics while a minority of populations have one or more 
metacentric chromosomes. Natural populations with one or more metacentric chromosomes have not been reported in other subspecies of $M$. musculus nor in closely related species ( $M$. spretus, M. spicilegus and M. macedonicus) -all of which have $2 \mathrm{~N}=40$ telocentric $X$ and autosomes (Garagna et al. 2014).

Although the empirical evidence for the karyotypic diversity of $M . m$. domesticus is well documented (e.g., Pialek et al. 2005), the evolutionary forces that that generate and maintain this diversity are unresolved. One clue comes from studies of centromere drive in this subspecies (reviewed in Lampson and Black 2017; Chmatal et al. 2017). These studies found that: i) populations with metacentrics have very small centromeres and small kinetochores compared to all-telocentrics populations, and ii) chromosomes with very small centromeres have a centromere drive disadvantage. A second clue to the causation for this extreme karyotype variation is the finding that the centromeric DNA repeat arrays (hereafter centromeres) of M. m. domestics have an unusual pattern of sequence variation. On a global scale, centromeric monomers show only moderate levels of divergence form the consensus sequence (averaging around 4\%; Kipling et al. 1994; Kalitsis et al. 2006; Rice 2020). Yet despite this substantial similarity to the consensus, sequence diversity of centromeric monomers is extremely high in other metrics (Rice 2020): high richness (many hundreds of sequence variants) and high evenness (no sequence variant is common).

Here I combine information from many disparate sources to describe and support a new evolutionary model for: i) high sequence diversity at the local level among the centromeric monomers, and ii) coevolution between centromere size and karyotype number. The key feature that underlies the operation of both models is a strong influence of telomeric proteins (from the shelterin complex) on the repair of DNA double strand breaks (DSBs) within centromeres when they are positioned near the end of a chromosome.

\section{Contributing factors for the hypotheses}

The models I develop for high monomer diversity and coevolution between centromere size and karyotype number combine diverse information from many seemingly unrelated areas. These include:

- Centromeric sequence variation at a local scale

- DNA repair near telomeres

- Expansion and contraction of centromeric repeat arrays

- Adaptive and maladaptive centromere drive

- Relationship between centromere size and kinetochore size

- Chromosomal fusions, fissions, Whole Arm Reciprocal Translocations (WARTs) and Sub-Telomere End Reciprocal Translocations (STERTs)

- Tubulin depolymerases

- The direction of centromere drive for different forms of de novo telocentrics and metacentrics

In the following sections, I summarize the relevant information in these areas (and some interactions between them). After these summaries, I will integrate the information in two model sections.

\section{Unusual pattern of monomer diversity at centromeric repeats}

In the companion paper (Rice 2020) I found the monomers of the centromeres of $M . m$. domesticus to have unexpected structure. When I randomly sampled monomers (120 bp) from 150 bp Illumina whole genome shotgun reads (with low sequencing error), I found an average divergence of $\sim 6 \%$ between random pairs of monomers (similar to previous estimates of this parameter; e.g., Kalitsis et al. 2006). In sharp contrast, the monomers of the centromeric repeats of humans commonly differ by more than $20 \%$ (for a summary of the consensus sequences of all monomers across all chromosomes from a single human genome see Supplemental Table 1 and Supplemental Figure S16 in Rice 2019A). For this reason, the monomers of $M . m$. domesticus have commonly been referred to as having low diversity. However, despite the moderate average level of sequence divergence between random pairs of mouse monomers, sequence diversity is high when measured in other ways: i) there are many hundreds of different sequence variants, indicating high numerical diversity $=$ high 'richness' (on average a new sequence was observed every four additional monomers 


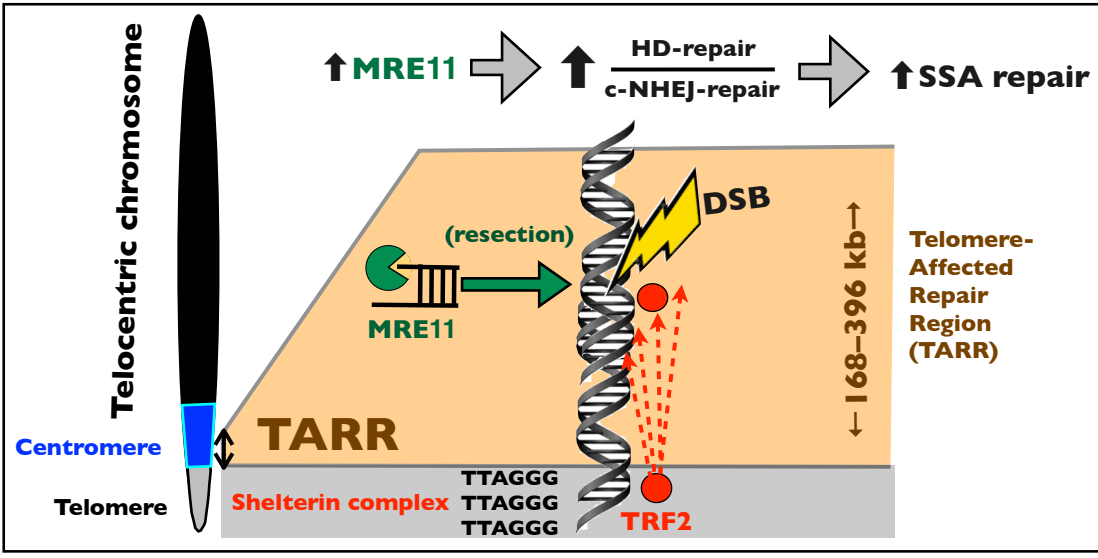

Figure 1. Influence of the Telomere-Affected Repair Region (TARR) on DNA repair. Chromosome ends (containing a DNA array of telomeric repeat units: TTAGGG) resemble one side of a DSB and must be shielded from DNA repair pathways (including both canonical non-homologous end joining (c-NHEJ) and homology-directed repair [HD-repair]). This shielding is accomplished by the recruitment of the shelterin complex of proteins to the telomeres. The region of DNA immediately adjacent to the telomeres (estimated to be 168-396 kb in humans) is exposed to elevated levels of the shelterin complex proteins, including TRF2. MRE11 is recruited by TRF2 and causes resection at DSBs, which in turn initiates HD-repair. This recruitment decreases the the proportion of DSBs repaired by c-NHEJ. Within tandem repeat arrays, an elevated rate of HD-repair is expected to increase the level of repair via the SSA pathway (that typically erodes one repeat unit per DSB). This figure is based on a series of papers exploring the influence of chromosome position on DNA repair of DSBs (reviewed in Muraki and Murnane 2017).

randomly sampled), ii) all sequence variants are rare with no predominant forms (indicating high uniformity in variant frequencies $=$ high 'evenness') iii) sequence diversity among small 'neighborhoods' of contiguous monomers is nearly as high as global diversity among all autosomes and the $X$, and iv) side-by-side monomers rarely share the same sequence (see the companion paper [Rice 2020] for details). In the models for high monomer diversity and coevolution between centromere size and karyotype number (described in later sections), I motivate the hypothesis that non-matching sequences among local 'neighborhoods' of centromeric monomers are selectively favored by molecular drive (Dover 1982) because this sequence heterogeneity reduces the deletion rate from repair of DSBs via the Single Strand Annealing (SSA) pathway.

2. A telomere-proximate position of centromeres is expected to influence DNA repair
In the model for coevolution between centromere size and karyotype number, the chromosomal position of the centromere evolves -so here I explore the relevant consequence of centromere position. As described more fully below, the chromosomes of M. m. domesticus are of two types: i) telocentrics, in which the telomere and the centromere are closely positioned, and ii) metacentrics, in which these structures are separated by a substantial distance. The location of the centromere along a chromosome is expected to influence the repair pathway by which double strand breaks (DSBs) are repaired.

A series of papers from the Murnane laboratory on laboratory mice (Lo et al. 2002) and humans (e.g., Zschenker et al. 2009 ; Kulkarni et al. 2010; Silva et al. 2017; reviewed in Muraki and Murnane 2017) demonstrated that chromosomal regions proximate to the telomere have unusual DNA repair. Chromosome ends (composed of telomeric repeat arrays) are protected from being recognized (and repaired) as one side of a DSB by the shelterin complex of proteins (see for review, de Lange 2018). By engineering ectopic endonuclease cleavage sites to be varying distances from the telomere, Murnane's group showed that 'excess processing' (more resection of 5' ends of DSBs) occurred when the sites were within a few hundred $\mathrm{kb}$ of the telomere (Figure 1). Throughout the following sections, I will refer to the telomere-proximate region of a chromosome that has increased levels of DNA resection during the repair of DSBs as the Telomer-Affected Repair Region (TARR). The increased level of DNA resection within TARR reduced the ratio of repair of DSBs by canonical Non-Homologous End-Joining (c-NHEJ) compared to Homology-Directed repair (HDrepair). Several lines of evidence indicated that this increased resection of DSBs was caused 


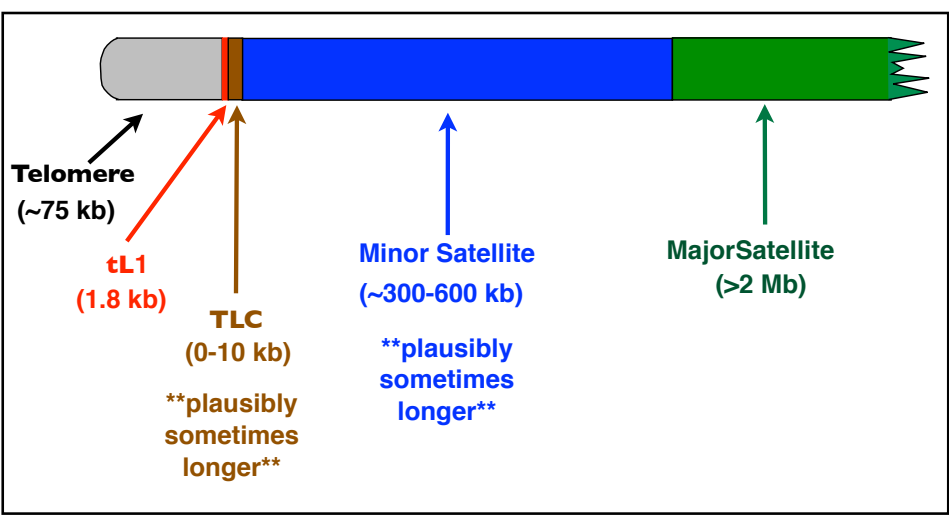

Figure 2. A schematic diagram of the organization of the subtelomeric region of the telomeric chromosomes of the Western European house mouse (centromere-containing end). The telomeric repeat array at the end of a telocentric chromosome of $M$. $\mathrm{m}$. domesticus $(\sim 75 \mathrm{~kb}$; the mid-range value from Thilagavathi et al. 2013) is flanked by a truncated L1 transposon $(\mathrm{tL} 1,1.8 \mathrm{~kb})$. Moving inward, the next block of DNA is an array of the TLC repeat (when present). This array varied in length from $0-10 \mathrm{~kb}$ in a sample from four chromosomes. Moving further inward, the next block of DNA is the minor satellite (centromeric repeat array). It was estimated to be $300-600 \mathrm{~kb}$ from the data of Kipling et al. (1991) and Aker and Huang (1996), but a recent genome-wide estimate based on read-depth was an average value of $716 \mathrm{~kb}$ (Iwata-Otsubo et al. 2017). The inner-most block of DNA is the pericentromeric heterochromatin (major satellite array, > $2 \mathrm{Mb}$; Kuznetsova et al. 2006). This figure is based on data from Kalitsis et al. (2006) that analyzed fosmid clones from the mouse genome project (mouse strain C57BL/6J).

primarily by the shelterin complex protein TRF2 which is concentrated at telomeric repeat DNA (Figure 1). TRF2 was shown to influence DNA repair at sites flanking the telomere by recruiting MRE11 which resects DNA ends generated by DSBs (Figure 1).

Repair of DSBs within centromeric (minor satellite) and pericentromeric (major satellite) repeat arrays was compared by Tsouroula et al. (2016) -see Figure 2 for the location of the major and minor satellites on telocentric chromosomes of $M$. $m$. domesticus. They selectively introduced DSBs in each satellite separately via CRISPERCas9 targeting of their consensus sequences. Quantitative measures of the size of the TARR in humans indicated that it extends over at least 100 $\mathrm{kb}$ with an estimated range of 168-396 kb (Muraki and Murnane 2017). The range may be higher in mice due to the fact that their telomeres are $5 x$ to 10x longer (Calado and Dumitriu 2013). The close proximity of the minor satellite to the telomere and the more distal location of its flanking major satellite (Kalitsis et al. 2006; see also Figure 2) would be expected to place much of the minor satellite within the Western European house mouse's TARR and all or most of the major satellite outside this region (Figure 2). Correspondingly, Tsouroula et al. (2016) found evidence for resection and HD-repair of DSBs throughout the cell cycle at the minor satellite arrays whereas for the major satellite arrays HD-repair was restricted to late $S$ and $G_{2}$ part of the cell-cycle (the pattern typical for most of the genome; Ceccaldi et al. 2016) (Figure $3)$. These researchers also found evidence that some of the HD-repair occurred via the SSA pathway, which is expected to produce deletions of one repeat unit per DSB repair event (e.g., see Ozenberger et al. 1991; Muchova et al. 2015; Bhargava et al. 2016; see also supplemental Figure S9 in Rice 2019B).

Repeated SSA repair of DSBs within the centromeric repeat array is expected to lead to a gradual erosion in its size and require some counterbalancing regenerative process (see Rice 2019B) as occurs in rDNA repeats (reviewed in Kobayashi \& Ganley 2005). The effect of TARR in amplifying the rate of SSA repair of DSBs would be expected to be eliminated when telocentric chromosomes fuse to form metacentrics -and this karyotypic change would be expected to slow the rate of erosion in minor satellite length due to SSA repair. Such a telocentric/metacentric difference in erosion of the minor satellite will be an important contributing factor in the model for the coevolution between centromere size and karyotype number.

\section{Expansion/contraction of centromeric repeat arrays}

In the model of coevolution between centromere size and karyotype number, the rates of expansion and contraction of the centromeric repeat arrays will be pivotal parameters. Here I summarize relevant information on these processes for centromeric DNA -and some of the consequences of the chromosomal position of the centromere.

The work by Tsouroula et al. (2016) described in the previous section indicates that low levels of 


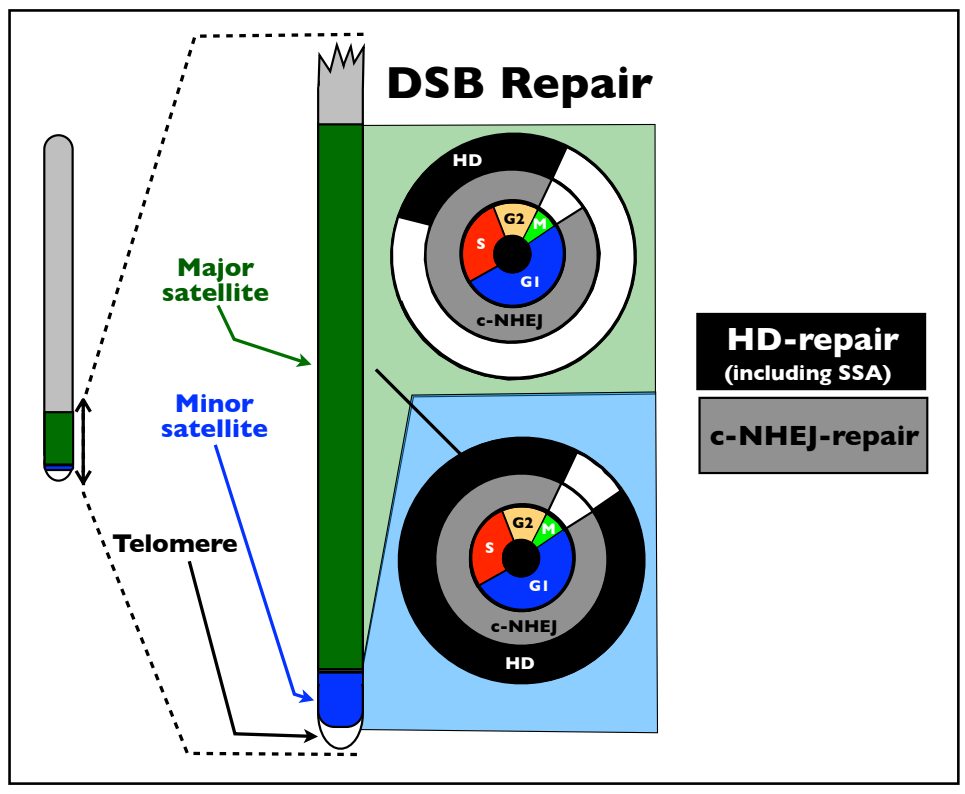

Figure 3. HD-repair of DSBs occurs at an elevated rate in the telomere-proximal centromeric DNA of $M$. $\boldsymbol{m}$. domesticus. DSBs in the DNA of the centromere (minor satellite) and heterochromatic pericentromere (major satellite) are repaired by both the c-NHEJ and HD-repair pathways. Repair via c-NHEJ has the same pattern of use across the cell cycle in both centromeric and pericentromeric DNA (cell cycle shown by the colored, interior circles in the figures). HD-repair, however, is more broadly used across the cell cycle in the centromere compared to the pericentromere. This figure is based on data from Tsouroula et al. (2016).

SSA repair of DSBs occurs throughout the cell cycle in sub-telomeric centromeric repeat arrays (minor satellites) of M. m. domesticus. Over time, this process is predicted to gradually erode the length of the centromeric repeat array (Ozenberger et al. 1991; Muchova et al. 2015; Bhargava et al. 2016) -ultimately reducing it to a sub-functional size (Lo et al. 1999; Yang et al. 2000; Okamoto et al. 2007). Unequal crossing over within the minor satellite is a process that can increase the length of a tandem array (Smith 1976), but this is a length-neutral process between a pair of repeat arrays that symmetrically both shortens one array and lengthens the other array to the same degree. As a consequence, unequal crossing over can only counteract the erosion in minor satellite length by random genetic drift of the length variants it generates. Alternatively, unequal crossing over in combination with selection for a functional minor satellite length could maintain operational length. However, average minor satellite length varies by up to an order of magnitude across Western
European house mouse subpopulations (Chmatal et al. 2014; Iwata-Otsubo et al. 2017 ), indicating that some subpopulations are plausibly maintaining array sizes that are far greater than required for cellular functioning.

Previously I presented evidence indicating that human centromeric repeat arrays are being maintained at sizes far in excess of the minimum required for cellular functioning, and that these largerthan-needed arrays were generated by their continuous expansion via out-ofregister Break Induced Repair (BIR) after collapse of replication forks (Rice 2019A, 2019B). In yeast, this process is responsible for the regrowth of rDNA repeat arrays after they experience major deletions because BIR duplication events exceed BIR deletion events (reviewed in Kobayashi 2014). Collapsed replication forks are expected to be far more common within the centromeric repeat arrays (compared to most other genomic regions) because constitutively bound kinetochore proteins (CCAN) plausibly block replication fork progression in humans (Crosetto et al. 2013; Aze et al. 2016) as they are established to do at both the point centromeres of budding yeast (Greenfeder and Newlon 1992) and the regional centromeres of Canida albicans (Mitra et al. 2014). Such continuous expansion of centromeric repeat arrays by out-of-register BIR repair after the collapse of replication forks has the potential to off-set the continual erosion of these arrays by SSA repair of DSBs. In M. $m$. domesticus, however, this potential is expected to vary between metacentric and telocentric chromosomes because in metacentrics SSA repair of DSBs is expected to be reduced because there is no TARR effect increasing the ratio of HD-repair/c-NHEJ, as would occur in telocentrics. This expected difference will play a key role in the model for the coevolution between centromere size and karyotype number.

\section{Influence of TARR on local-level diversity of centromeric monomers}

Exceptionally high sequence diversity of monomers (high richness and evenness) at a local level was observed in the companion paper 


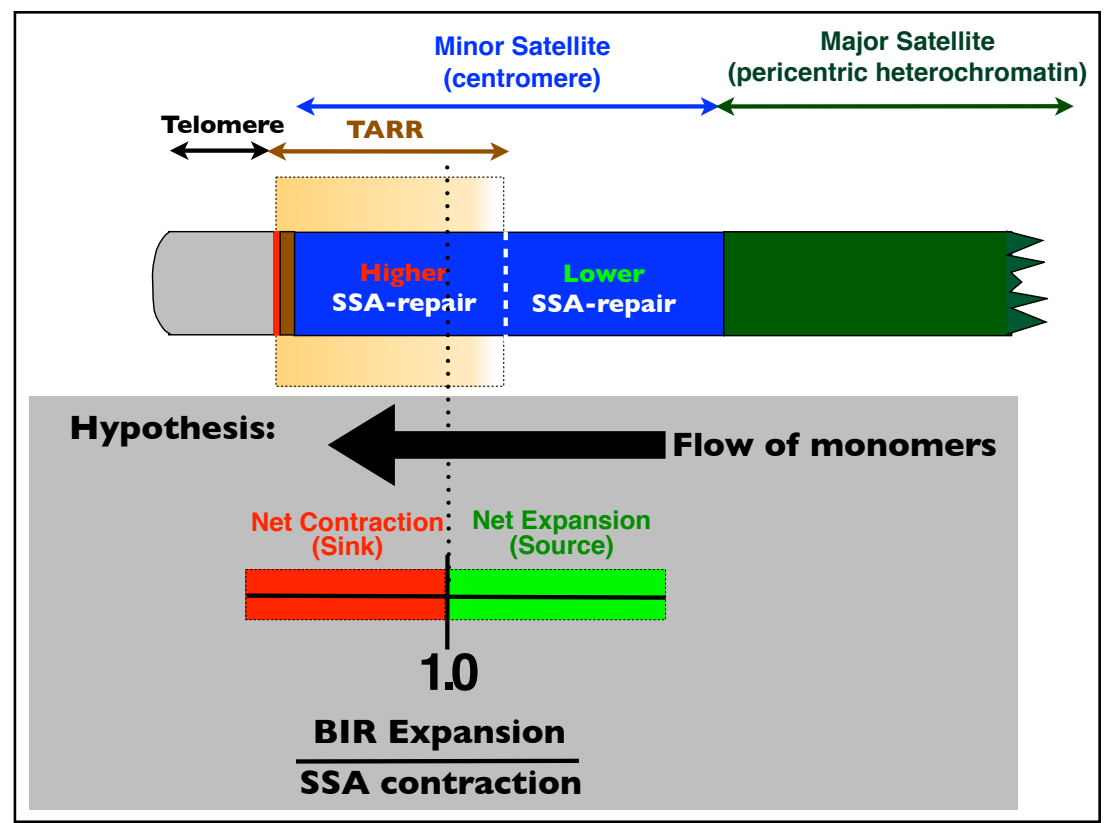

Figure 4. The spatial restriction of TARR is expected create sink/ source structure within centromeres. There are no published studies of the spatial range of TARR on the chromosomes of the Western European house mouse, but studies in human cells (reviewed in Muraki and Murnane 2017) suggest that it would plausibly span several hundred kb. The close proximity of the centromere to the telomere would therefore be expected to place at least part of the centromere within the TARR. Hypothesis: Assuming that regions of the centromere closer to the telomere experience a stronger TARR effect (higher rate of SSA deletions), and that the centromere is not expanding, then the centromere will necessarily be bisected into two parts: i) a telomere-proximate side (sink) that is contracting by SSA repair faster than expanding via BIR (depicted in bright green in the bottom of the figure), and ii) a telomere-distal side (source) that is expanding via BIR faster than contracting by SSA repair (red). This structure would generate constant turnover of monomers and a net flow of monomers from source to sink regions. The source/sink dichotomy and flow of monomers will more generally be realized whenever TARR causes SSA contraction to be larger than BIR expansion at the centromeric region closest to the telomere. When the sink region is too large relative to the source region, the centromeric repeat array (minor satellite) would be expected to shrink over time.

on the structure of centromeric monomers (Rice 2020). Here I summarize information on how SSA repair of DSBs, in combination with centromere position (via a TARR effect), is expected to generate selection for non-identical sequences among clusters ('neighborhoods') of centromeric monomers -but not requiring high levels of sequence divergence. I also speculate on how this small neighborhood of non-matching monomers also might be favored by BIR repair of collapsed replication forks.
SSA repair of DSBs generates selection (in the context of molecular drive; Dover 1982) on the sequence of neighboring centromeric monomers. More distant spacing between matching sequences reduces the efficacy of homology search (Wang et al. 2017), and small changes in DNA sequence (homeology) has a large negative effects on the homology recognition step of homologydirected DNA repair of DSBs (Waldman and Liskay 1988; Bhattacharjee et al. 2014). As a consequence, side-by-side or near-by monomers with identical sequence are more susceptible to deletion via SSA repair of DSBS than monomers without nearby identical sequence. This liability generates selection for neighboring monomers to diverge in sequence -especially within the TARR region where rates of SSA repair are elevated and resection tract lengths are longer (Muraki and Murnane 2017). Selection to avoid deletion during SSA repair only applies to identical monomers that are close enough to be included in the resected regions on either side of a DSB.

Although more speculative, stretches of non-matching monomers within a repeat array also might be favored by molecular-drive due to BIR repair of collapsed replication forks. Initiation of out-of-register BIR after fork-collapse would be expected to occur predominantly between monomers of identical sequence because only small deviations in sequence are required to substantially interfere with homology recognition (Waldman and Liskay 1988; Jain et al. 2016). As a consequence, larger duplications per out-ofregister BIR occur when monomers of identical sequence are more distantly separated (but not too distantly separated; Rice 2019B). This feature would be expected to generate selection (via molecular drive) for local neighborhoods of nonmatching monomers (that separate matching 
sequences) in the same way that it is hypothesized to select for longer HORs in humans (see Figure 10 of Rice 2019B for a fuller explanation). However, this intra-array expansion advantage is offset by a contraction disadvantage due to longer deletions per DSB via SSA repair, especially when resection tract lengths are long (see Rice 2019 [supplemental Figures S8 and S10] for a quantitative analysis of these tradeoffs). Quantification of this expansion advantage and contraction disadvantage are needed to determine if stretches of non-matching monomers are favored by a higher intra-array expansion rate.

In sum, deletion by SSA, and possibly expansion by BIR, are expected to generate molecular-drive selection for dissimilarity in sequence among close-by monomers. Consistent with this selection, in the companion paper (Rice 2020) side-by-side monomers rarely matched in sequence and only a low frequency of monomers with matching sequence was found in the neighborhoods of 5 contiguous monomers in the centromeric repeat arrays of $M$. m. domesticus.

\section{Telomere proximity is expected to generate a flow of monomers from expanding "source" to contracting "sink" regions within a centromere}

The models for high monomer diversity and coevolution between centromere size and karyotype number predict that monomers chronically turn over within centromeres because they are dynamic: continually being duplicated by out-of-register BIR after replication-fork collapse, and continually being deleted by SSA repair of DSBs. When the centromere is close to the telomere, the duplication/deletion ratio is expected to vary with distance from the centromere and generate a net flow of monomers from the telomere-distal region of the centromere (source) to the telomere-proximate region (sink). Next, I develop the rationale for this hypothesized flow of monomers within the centromeres of telocentric chromosomes.

Sequence analysis of the p-arms of a sample of telocentric chromosomes from $M$. m. domesticus indicated that the centromere can reside very close to the telomere $(<2 \mathrm{~kb}$, Kalitsis et al. 2006), as illustrated in Figure 2. This close proximity indicates that at least part of a typical mouse centromere (spanning 300-600 kb; Komissarov et al. 2011 -but see Iwata-Otsubo et al. [2017] for evidence of a larger average size of $716 \mathrm{~kb}$ in strain C57BL/6N) would be expected to be within the TARR (Figure 4). Inclusion within the TARR is expected to increase repair of DSBs via the HDrepair pathways, including SSA repair (Figure 4; Muraki and Murnane 2017). Increased use of the SSA repair pathway would increase the level of centromere contraction (via deletions) compared to to centromere expansion (via duplications from out-of-register BIR after replication-fork collapse), i.e., reduce the BIR-expansion/SSA-contraction ratio (Figure 4).

One of the hallmarks of TARR is "excess processing" in which: i) the frequency of HDrepair of DSBs is increased (compared to repair via c-NHEJ), and ii) the length of the $5^{\prime}$ resected regions is expanded substantially (reviewed in Muraki and Murnane 2017). These two features would be expected to increase the contraction rate of the centromere because: i) SSA repair is more common, and ii) longer deletions would occur because regions of close homology that are more distantly separated would more commonly be uncovered by resection and used during SSA repair. The increase in contraction rate would be expected to be maximal at locations closest to the telomere.

If the centromere is not increasing in size over time, then the telomere-proximate region of the centromere will have an excess of deletions over expansions and vice versa for the telomere-distal region. This polarity motivates the hypothesis that the much of the region of the centromeric repeat array within the TARR (that is sufficiently close to the telomere) is contracting (SSA contraction > BIR expansion) while locations outside this region are expanding (SSA contraction < BIR expansion) -see Figure 4. As a consequence, the centromeric repeat array is divided into two regions (Figure 4): i) a telomere-proximate "sink" region where monomers are being deleted by SSA faster than they duplicate via BIR, and ii) and a telomere-distant "source" region where BIR duplicates monomers faster than SSA deletes them. This sink/source dichotomy would be expected to generate a net flow of monomers from the expanding telomere-distal region of the centromere into the contracting telomereproximate region (Figure 4). 


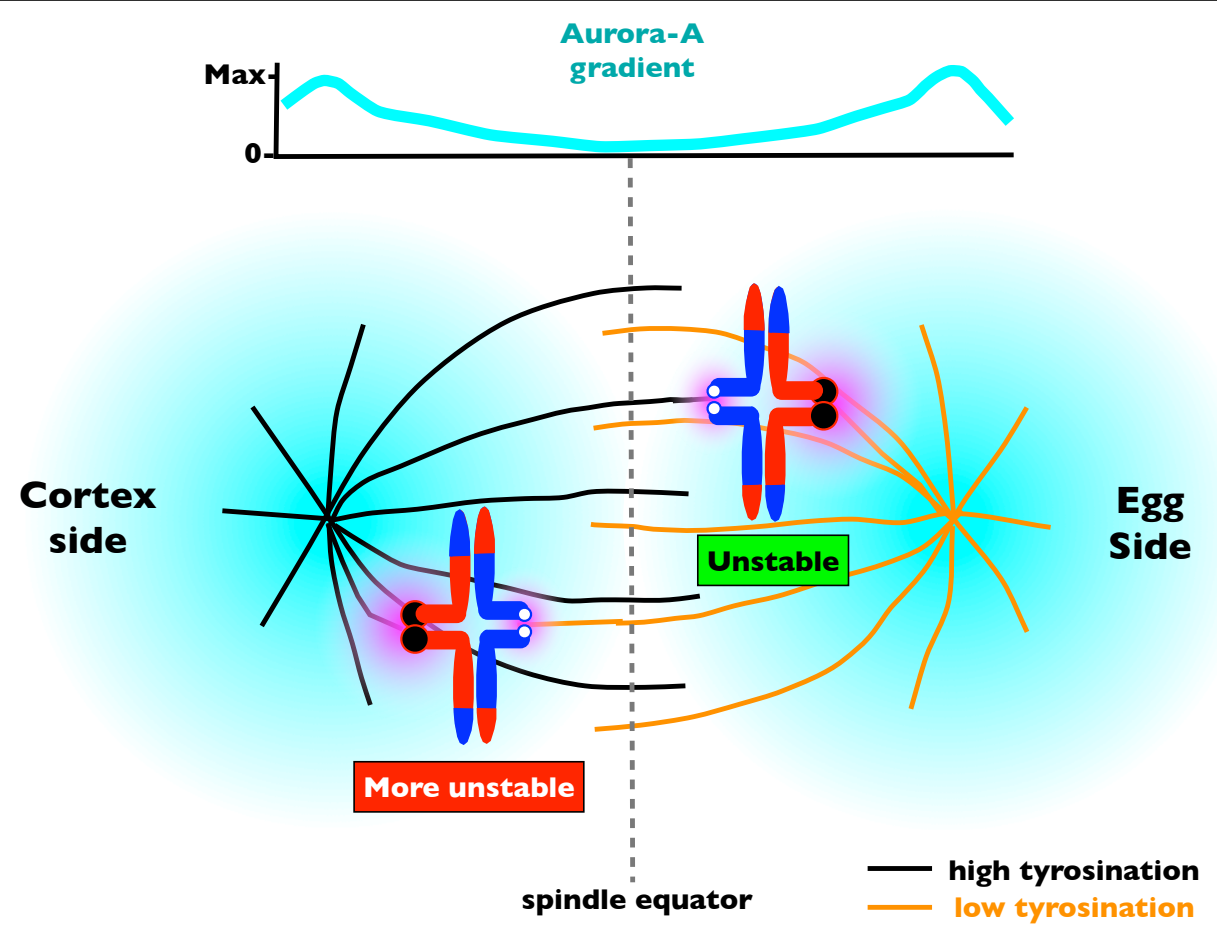

Figure 5. Asymmetry in both spindle tyrosination and kinetochore recruitment of MCAK generates off-center bivalents and centromere drive. The figure depicts two alternative positions of the same bivalent during late metaphase-I of meiosis. The positions are determined by random initial alignment when the bivalent orients with its larger pair of kinetochores pointing toward the cortical (left) or egg (right) side of the spindle. The two homologous pairs of sister chromatids within the bivalent are depicted as red or blue L-shaped threads, and color-switching within a thread depicts a crossover. The homologs have centromeres that differ in size: blue chromatids have very small centromeres and red chromatids have normal-sized centromeres. The difference in centromere size generates asymmetry in kinetochore size: blue chromatids with very small centromeres recruit small kinetochores (white circles) and red chromatids with normal-sized centromeres recruit normal-sized kinetochores (black circles). Asymmetry in kinetochore sizes generates poleward-displacement of the bivalent (an off-center position toward the pole facing the larger pair of kinetochores) because small kinetochores recruit lower levels of tubulin depolymerase (MCAK; graded purple color; Akera et al. 2019). This displacement is plausibly generated by asymmetric pulling force of spindle fibers via the pacman process (reviewed in, Cross and McAnish 2014; Kent and Lee 2017; see Figure 6) due to the differences in MCAK recruitment -but this hypothesis has not been experimentally tested. Displacement of the bivalent off the spindle equator causes the bivalent to experience elevated concentrations of the kinase Aurora- $A$ (graded turquoise color and the upper graph) which destabilizes kinetochore-spindle connections and thereby increases detachment rate. The detachment rate is higher on the cortical side of the spindle (that will give rise to the polar body) because it is more highly tyrosinated: which increases the tubulin depolymerase-induced detachment rate of kinetochores from the spindle; (Peris et al. 2009 ; Sirajuddin et al. 2013; Akera et al. 2019). Because an off-center bivalent has elevated kinetochore/spindle detachment rates, it is unstable (loses spindle attachment) and reorients (relative to the two spindle poles) at elevated rates compared to on-center bivalents containing homologs with similar centromere/kinetochore sizes. In addition, because detachment is elevated more on the more highly tyrosinated (cortical) side of the spindle, the bivalent is more unstable (detaches from spindle more frequently) when oriented with the smaller centromere/kinetochore facing the egg side of the spindle: leading to centromere drive against the homolog with the very small centromere and small kinetochore. This figure is based on my interpretation of data and information from Chmatal et al. (2015), Akera et al. (2017) and Akera et al. (2019).

In the coevolution model section of this paper I will explore the consequences of TARR-induced contraction of centromeric repeat arrays because (as described below) it is a feasible explanation for the empirical observation that some populations of $M . m$. domesticus have evolved extremely small centromeres that lose in centromere drive (segregate to the polar body more than $50 \%$ of the time) when introgressed into laboratory populations with normal 


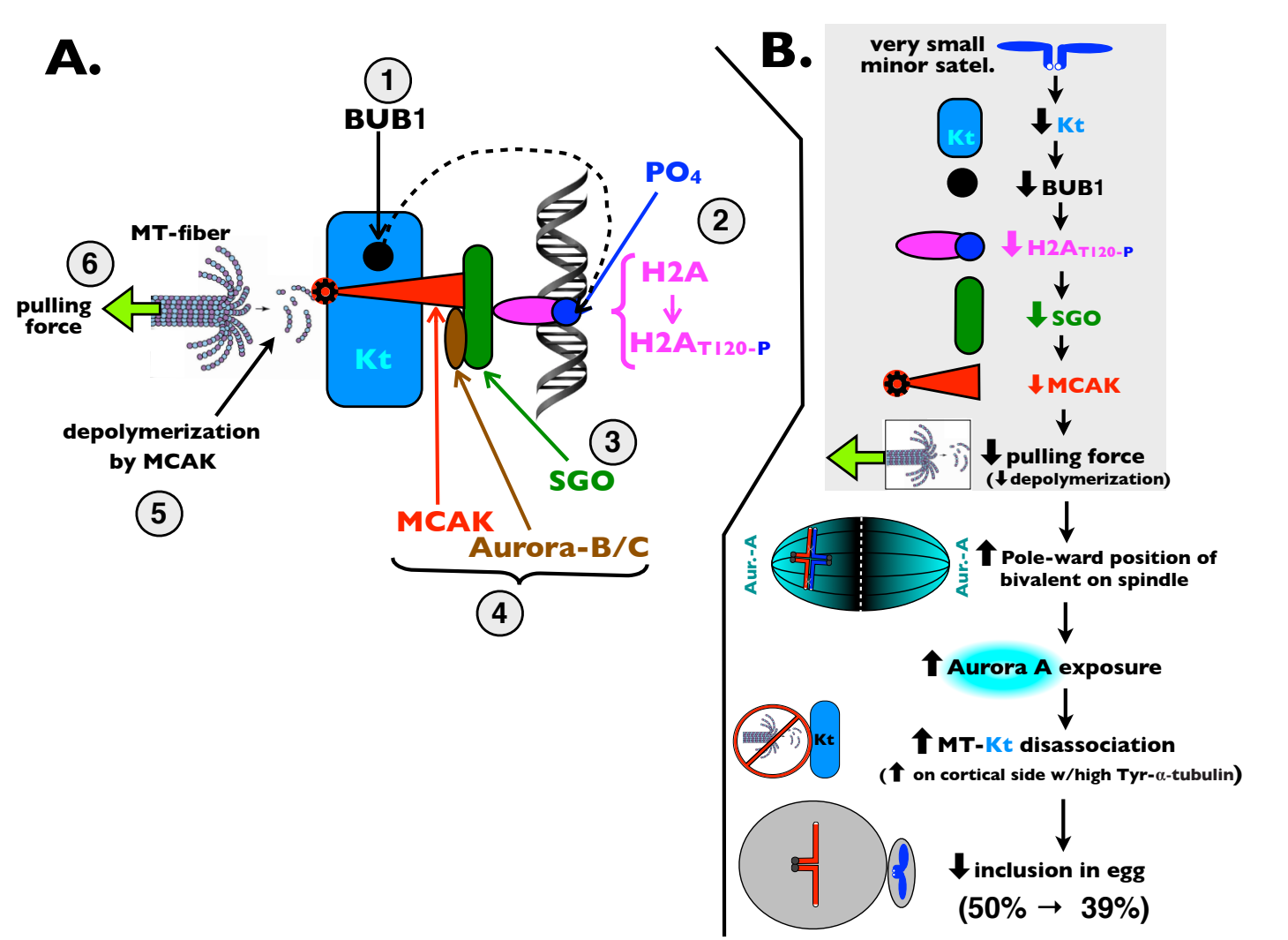

Figure 6. The cascade of molecular events leading to late-acting centromere drive that favors homologs with larger kinetochores in $\mathbf{M}$. $\boldsymbol{m}$. domesticus. A. 1. Larger kinetochores recruit more of the histone kinase BUB1. 2.

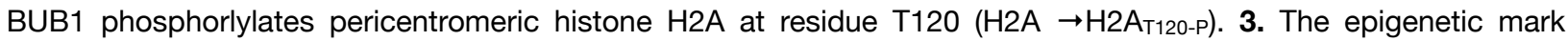
H2A $A_{120-P}$ recruits Shugoshin (SGO). 4. SGO acts as the scaffold for Aurora-B and C kinases and MCAK. 5-6. The tubulin depolymerase activity of MCAK is hypothesized to generate pulling force via the Pacman process. B. The molecular steps linking small kinetochore size to a disadvantage in centromere drive. This figure is based on data and information from Akera et al. (2017) and Akera et al. (2019).

centromere sizes (Nachman and Searle 1995; Iwata-Otsubo et al.2017). When the sink region is too high of a proportion of the entire centromere, the centromere will shrink over time. Shrunken centromeres, however, plausibly can be rescued by a combination of chromosome fusions and centromere drive, as described in later sections.

\section{Centromere drive in $M$. m. domesticus can be both adaptive and maladaptive}

Adaptive centromere drive plays a pivotal role in the model for coevolution between centromere size and karyotype number. Here I summarize recent research on one form of centromere drive that is adaptive and another form that is maladaptive.

Two forms of centromere drive in $M . m$. domesticus are described in Box-1. The first form operates during early metaphase- 1 of meiosis (before spindle migration to the cortex). In this drive system, the homolog with a major deletion in the pericentric heterochromatin (pericentromere or major satellite array ) is favored by centromere drive. The second form operates late in metaphase- 1 of meiosis (after spindle migration to the cortex). In this drive system, the homolog with an exceptionally small centromere (minor satellite array) is disfavored by centromere drive. The two centromere drive systems have fundamentally different molecular foundations and evolutionary consequences. The late-acting centromere drive is based on asymmetric tyrosination of the two halves of the spindle in combination with asymmetry in kinetochore size between homologs (which depends on a difference in size between homologous centromeres). The early-acting centromere drive is based on asymmetric tubulin levels of the two halves of the spindle in combination with asymmetry in the size of the pericentromere between homologs. In one case 


\section{Box 1. The Western European house mouse has two distinct forms of meiotic drive.}

Late-acting drive: Beginning with the work of Chmatal et al. (2014), a succession of studies from the Lampson laboratory have made substantial progress in our understanding of one form of centromere drive in $M$. m. domesticus (Chmatal et al. 2015; Akera et al. 2017; Iwata-Otsubo et al. 2017; Akera et al. 2019). The cellular asymmetry that lies at the foundation of this lateacting centromere drive is the level of tyrosination of alpha-tubulin in spindle fibers (Figure 5): tyrosination is much higher on the side of the spindle that will give rise to the polar body (the cortex side) -but only for a few hours immediately preceding the onset of anaphase-I of meiosis. Tyrosination of alpha-tubulin influences the activity of the tubulin depolymerase MCAK (aka Bif2C), which is recruited to the two paired kinetochores that attach a bivalent to the spindle during early metaphase-I of meiosis (Figure 5). MCAK activity, in combination with bivalent position on the spindle, influences kinetochore detachment from the spindle (Figures 5 and 6) -a key feature in the process of centromere drive.

Kinetochore detachment is regulated in part by the depolymerase activity of MCAK and this activity is enhanced by tyrosination of alpha-tubulin: more tyrosination $\rightarrow$ more depolymerization by MCAK $\rightarrow$ higher rate of detachment of kinetochores from their spindle-fibers (Peris et al. 2009; Sirajuddin et al. 2013; Akera et al. 2019). Kinetochore detachment prior to anaphase-I of meiosis is also strongly influenced by the position of a bivalent on the spindle because location determines exposure to Aurora-A -a kinase that destabilizes kinetochore/spindle-fiber attachments (Figure 5). When a bivalent is displaced off the spindle equator toward a pole, kinetochores experience a higher concentration of Aurora-A kinase (which is concentrated near the spindle poles; Figure 5) $\rightarrow$ a higher detachment rate between kinetochores and their spindle-fibers (the closer to the pole the higher the detachment rate; Chmatal et al. 2015).

Chromosomes with very small centromeres lose in centromere drive (segregate to a polar body more than $50 \%$ of the time) because they recruit smaller kinetochores (reviewed in Lampson and Black 2017; Chmatal et al. 2017). The molecular mechanism connecting small centromere size and losing in centromere drive is illustrated in Figures 5 and 6 . By a series of intermediate steps (described in Figure 6B), very small centromeres recruit smaller kinetochores that recruit less MCAK tubulin depolymerase -compared to normal-sized kinetochores that are recruited to normal-sized homologous centromeres. This asymmetry between a normal sized pair of kinetochores and a small pair causes a bivalent to be positioned off-center and closer to the spindle poles facing the larger pair of kinetochores (Figure 5).

Off-center positioning of a bivalent leads to an Aurora-A-induced elevated rate of kinetochore/spindle-fiber detachment (unstable spindle attachment) and subsequent random realignment of the bivalent on the spindle (Chmatal et al. 2015). Because offcenter bivalents have i) a higher spindle detachment rate on the tyrosinated side of the spindle, and ii) an orientation with the smaller pair of kinetochores pointing away from the closer spindle pole, they are are more stable when the pair of sister chromatids with smaller centromeres/kinetochores is facing the cortex (Figure 5). As a consequence, off-center bivalents are more commonly aligned to segregate the smaller kinetochores to the polar body (Figure 5). These features cause the pair of sister chromatids with very small centromeres -and resulting small kinetochores- to segregate to the polar body more than $50 \%$ of the time -and thereby lose in centromere drive.

Asymmetric bivalents (with small and normal-sized kinetochores) expressed elevated rates of kinetochore/spindle-fiber detachment only when: i) they were positioned off-center of the spindle equator and, ii) Aurora-A was present at normal levels (i.e., not experimentally down-regulated; Chmatal et al. 2015). These results indicate that two key features generating centromere drive against chromosomes with very small centromere are: i) decreased recruitment of MCAK to the pair of smaller kinetochores (that causes off-center positioning of a bivalent), and ii) increased exposure of the bivalent to Aurora-A kinase (that causes higher detachment rates between kinetochores and their spindle-fibers). An additional key feature is increased MCAKinduced detachment between kinetochores and their spindle-fiber on the more highly tyrosinated cortical side of the spindle.

Throughout this paper I will assume that lower recruitment of MCAK by one of the two pairs of kinetochores within a bivalent generates an asymmetry in poleward movement when a bivalent attempts to line-up on the metaphase plate prior to the completion of metaphase-I during meiosis (Figure 5). One underlying cause of this difference in poleward movement might be differences in pulling force. Pulling force was not measured in the study by Akera et al. (2019) but they did show that MCAK asymmetry at kinetochores within bivalents is associated with their off-center position on the meiotic spindle during metaphase-I.

An intuitive hypothesis for the off-center positioning of bivalents with asymmetric kinetochore sizes is asymmetric pulling force on the kinetochores. This hypothesis is supported by a series of observations. First in vitro experiments show that depolymerization of a microtubule by MCAK generates force that pulls glass beads behind the MCAK as it shortens a microtubule (Oguchi et al. 2011). Second, MCAK is the primary tubulin depolymerase that is active at kinetochores during metaphase of mitosis (Bakhoum et al. 2009; see also Supplemental Figure S6), and it is also plausibly the primary tubulin depolymerase acting on bioriented bivalents as they move toward alignment on the spindle equator during metaphase-I of meiosis. 


\section{Box-1 (continued)}

Third, depolymerization of spindle-fibers is experimentally established to be the primary force pulling kinetochores toward spindle poles via the Pacman process (reviewed in, Cross and McAnish 2014; Kent and Lee 2017). Fourth, asymmetric recruitment of MCAK to the kinetochores of a bivalent displaces the bivalent off the spindle equator and toward the pole facing the kinetochore with more MCAK - and experimentally equalizing MCAK recruitment removes this displacement (Akera et al. 2019). Collectively, these observations support the hypothesis that asymmetric pulling force is responsible for the off-center position of bivalents during late-acting centromere drive. Nonetheless, some other process may underly the offcenter positioning of these bivalents that recruit differing levels of MCAK between homologs. What is clearly established is that asymmetric kinetochore size leads to asymmetric poleward displacement of bivalents -toward the pole facing the larger kinetochore.

Early-acting drive: A second form of centromere drive in M. m. domesticus was recently described by Wu et al. (2018). Unlike the late-acting centromere drive described above, winning chromosomes in this drive system were estimated to have kinetochores that were $\sim 30 \%$ smaller than normal. In addition, this second form of centromere drive operates before spindle migration to the cortex of the egg, i.e., before the development of asymmetric tyrosination of the two halves of the spindle.

The DNA feature that underlies early-acting centromere drive was not unambiguously determined by Wu et al. (2018). They analyzed two driving chromosomes they labelled * and **. Both had major deletions in the pericentric heterochromatin (i.e., the major satellite array flanking the centromere: $83 \%$ deleted for ${ }^{*}$ and $96 \%$ deleted for $\left.{ }^{*}\right)$ and both had larger than average centromeres (i.e., minor satellite: $280 \%$ larger for ${ }^{*}$ and $160 \%$ larger for ${ }^{* \star}$ ). Because the estimated level of drive was slightly larger for the ${ }^{* \star}$ chromosome which had the slightly larger deletion of the major satellite (3.5 driver to 1 wild type for ** and 3.2 driver to 1 wild type for ${ }^{*}$ ) despite having a much smaller minor satellite, the drive-inducing feature is most plausibly the large deletion in the major satellite. This interpretation is supported by the finding that other mouse chromosomes with deleted major satellites (C-bands) have also been reported to produce transmission ratio distortion (Marciniak et al. 2010). For these reasons, in the remainder of this section I will assume that the large deletion in the major satellite is most plausibly the causative-feature for centromere drive in this system.

The centromere drive system analyzed by Wu et al. (2018) is based on a more subtle spindle asymmetry in early metaphaseI of meiosis (compared to the tyrosination asymmetry observed in late metaphase-I for late-acting centromere drive): one side of the spindle (the one that will later become the cortical side after spindle migration) has slightly more tubulin and generates substantially greater pull on kinetochores (see Figure 7A for quantitative details). This tubulin asymmetry had no measurable effect on wild type chromosomes (with average-sized major and minor satellites) but strongly influenced the stability of spindle-fiber/kinetochore attachment of the the driving ${ }^{*}$ and ${ }^{* *}$ chromosomes (Figure 7B and 7C). In what follows I will refer to cortical and egg sides of the spindle before spindle migration has occurred because Wu et al. (2018) have shown that the side with higher tubulin consistently migrates to the cortex.

Consider a heterozygous bivalent containing one pair of sister chromatids with average-sized major satellites (the wild type homologs) and the other pair of chromatids with a large deletion in the major satellite (the mutant homologs). Wu et al. (2018) found that when the mutant centromeres are oriented toward the egg side of the spindle (weaker pulling) they rarely detach from their spindle-fiber (no cases were observed) and therefore rarely changes orientation (i.e., they rarely reverse polarity; Figure 7C). But when the mutant centromeres are oriented toward the cortical side (stronger pulling), they detach and reverse polarity about half the time (Figure $7 \mathrm{C}$ ). This asymmetrical reversal of polarity across the two initial (random) orientations of the bivalent causes the driver to eventually orient toward the egg $\sim 75 \%$ of the time -thereby generating strong centromere drive (about three-to-one for segregation to the egg vs. the polar body, respectively).

The biological basis for the higher reversal of polarity when the driving chromosome faced the cortex side of the spindle is unknown. One intuitive hypothesis is based on the physical properties of the major satellite that makes up the pericentric heterochromatin: this chromatin has spring-like properties (Bouck and Bloom 2007; Stephens et al. 2013). During the chromosomal oscillations in early metaphase, tension is generated on the kinetochore/spindle-fiber connection. Wu et al. (2018) estimate that this tension (as measured by centromere-kinetochore separation) is substantially higher (up to $50 \%$ ) on the cortical side of the spindle (with higher tubulin levels; Figure 7A). If the pericentric heterochromatin acts as a spring-like shock-absorber, it would buffer the kinetochore/spindle-fiber connection from this tension and buffering would be greatly reduced on the mutant chromosome: causing it to detach more frequently when facing the cortical side of the spindle and thereby potentially reverse polarity toward the egg side. This asymmetry would generate the strong centromere drive observed by Wu et al. 2018. 


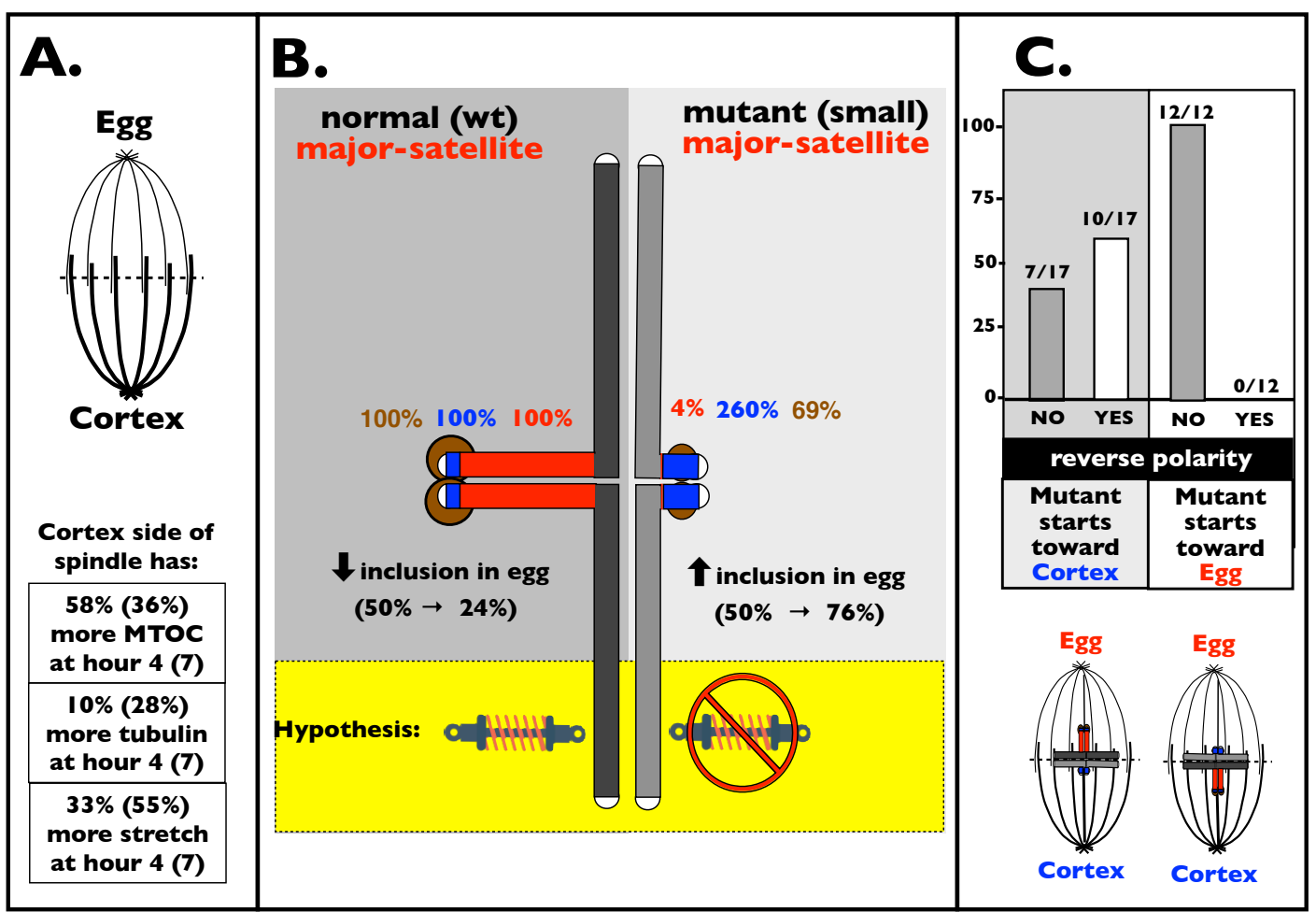

Figure 7. Early-acting centromere drive in $M$. $\boldsymbol{m}$. domesticus favors homologs with large deletions in the pericentric heterochromatin. A. Spindle asymmetry in the number of microtubule organization centers (MTOC), density of tubulin, and pulling force (as measured by the level of chromosome stretching). The box shows the excess of each parameter on the cortical compared to the egg side of the spindle. B. Comparison of chromosome structure between the wild type (on left, with a full-sized pericentromere -that loses in centromere drive) and mutant ( ${ }^{* *}$ chromosome on right with a grossly deleted pericentromere -that wins in centromere drive) homologs. Brown colored circles denote kinetochore size, red bars denote the size of the pericentric major satellite array, and blue bars denote the size of the centromeric minor satellite array. Colored percent values denote the relative size of the matching colored structure. The yellow box illustrates the hypothesis that the spring-like properties of the major satellite act as a shock absorber, which when deleted, causes an increased rate of spindle/kinetochore detachment when facing the the stronger-pulling cortical side of the spindle. $\mathbf{C}$. The mutant chromosome was not observed to detach from its spindle-fiber and reverse its polarity (change the spindle pole toward which it was facing) when it was initially facing the egg, but it frequently detached and reversed polarity when it was initially facing the more strongly-pulling cortical side of the spindle. Wild type chromosomes rarely detached and reversed polarity irrespective of their initial facing orientation. This figure is based on data and information from Wu et al. (2018).

meiotic drive is adaptive (late-acting -disfavoring an undersized centromere) and in the other case it is maladaptive (early-acting -favoring a grosslydeleted pericentromere).

There is a general consensus in published papers and books that centromere drive is maladaptive and is driven by selfish DNA (e.g., Henikoff et al. 2001; Burt and Trivers 2006; Rosin and Mellone 2017). However, as I have suggested previously (Rice 2019B), for hundreds of millions of years eukaryotes have begun meiosis with two homologous centromeres yet place only one of these in the functional egg. So, for eons there has been selection to favor egg-inclusion of the better centromere whenever one of the pair is functionally defective. The late-acting form of centromere drive selects against unusually small centromeres (with large deletions) that produce defective (small) kinetochores that recruit low levels of the tubulin depolymeriase MCAK. Asymmetric MCAK recruitment generates displacement of a bivalent away from the spindle equator and toward the pole facing the larger kinetochore (see Figure 5 and Box-1). In the model of coevolution between centromere size and karyotype number described in a later section, I will show how this phenotype can plausibly rescue populations of $M$. m. domesticus from chronic erosion of their centromeres. 


\section{Influence of repeat array size on kinetochore size and functioning}

The influence of centromere size on kinetochore size and performance is a key feature in the model of coevolution between karyotype number and centromere size. Here I summarize what is currently known about this interaction.

The relationship between kinetochore size and centromeric size has been studied in both mice and humans -but there is far more information in humans. In $M$. $m$. domesticus, the size of centromeres (centromeric DNA repeat arrays) was compared between 'winners' (> 50\% segregation to the egg) and 'losers' (> 50\% segregation to the polar body) in the context of centromere drive (Iwata-Otsubo et al. 2017). A strain with all loser chromosomes (CHPO) was found to have highly reduced centromere size (86-91\% smaller) compared to a laboratory strain (C57BL/6J) with all winner chromosomes. The average size of the loser centromeres was estimated to be only $83 \mathrm{~kb}$ (compared to $716 \mathrm{~kb}$ for the laboratory strain C57BL/6J). The kinetochores produced by these very small centromeres were substantially reduced in size: $\sim 40 \%$ smaller, as measured by the level of the kinetochore protein HEC1/NDC80 (levels of CENP-A and CENP-C were also reduced). Unfortunately, I have not found studies that report the influence on kinetochore size of less extreme differences in centromere size of $M . m$. domesticus.

In humans, Bodor et al. (2014) found the level of CENP-A (the foundational protein during kinetochore assembly) recruitment across all autosomes and $\mathrm{X}$ to be nearly uniform despite the fact that human genomes have high variation in centromeric array sizes between homologs and across chromosomes (e.g., Willard 1991, Miga et al. 2014). Bodor et al. (2014) also found that kinetochore size was little influenced by the CENP-A content at a centromere across a wide range of levels (levels of CENP-A between $40 \%$ and $240 \%$ of wild-type levels). Marzais et al. (1998) compared the kinetochore size on human chromosome 21 across centromeric array (alpha satellite) sizes that spanned more than an order of magnitude (0.42 $\mathrm{Mb}$ to $5.28 \mathrm{Mb}$ ). Despite this broad range in the size of the centromere, no detectable difference in kinetochore size was observed. Nonetheless, a very small size of a centromeric repeat array clearly has consequences. Analysis of exceptionally small centromeric repeat arrays in humans demonstrated that a minimum size is required for normal cellular functioning $(50-100 \mathrm{~kb}$; Lo et al. 1999; Yang et al. 2000; Okamoto et al. 2007).

These studies in humans collectively indicate that most natural variation in the size of centromeric repeat arrays has little or no effect on kinetochore size and functioning unless the array passes below a minimal threshold of very small size. This relation would plausibly also apply to $M . m$. domesticus, as I will assume in the model for the coevolution between centromere size and karyotype number.

\section{Sub-Telomere End Reciprocal Translocations (STERTs)}

Reciprocal translocations among the subtelomeric regions of the telocentric chromosomes of $M . m$. domesticus are an important feature in the model for coevolution between karyotype number and centromere size. Here I summarize the relevant terms and processes.

Consider the end of a telocentric chromosome that is nearest to the centromere. This subtelmeric region of $M . m$. domesticus (strain C57BL/6J) is shown schematically in Figure 2 (based on a study by Kalitsis et al. 2006). Immediately adjacent to the terminal telomeric repeat array is a truncated $\mathrm{L} 1$ transposable element (tL1). This feature is shared by all $M$. musculus strains and all subspecies that have been screened as well as two closely related species (M. spicilegus and M. spretus; Kalitsis et al. 2006). A short repeat array of the TLC sequence is usually found flanking tL1 (Figure 2). Although the TLC sequence has been detected in samples from Western European house mouse's three most closely related species $(M$. spicilegus, $M$. spretus, and $M$. caroli), it is absent in: i) more distantly related species, ii) some subspecies of M. musculus, and iii) some chromosomes within the genome of M. m. domesticus (Sasaki et al. 2013). Moving past the TLC repeat region and toward the interior of the chromosome, the next flanking sequences are the minor satellite (recruits the kinetochore), followed by the major satellite (recruits the pericentric heterochromatin and cohesin)(Figure 2). This subtelomeric 


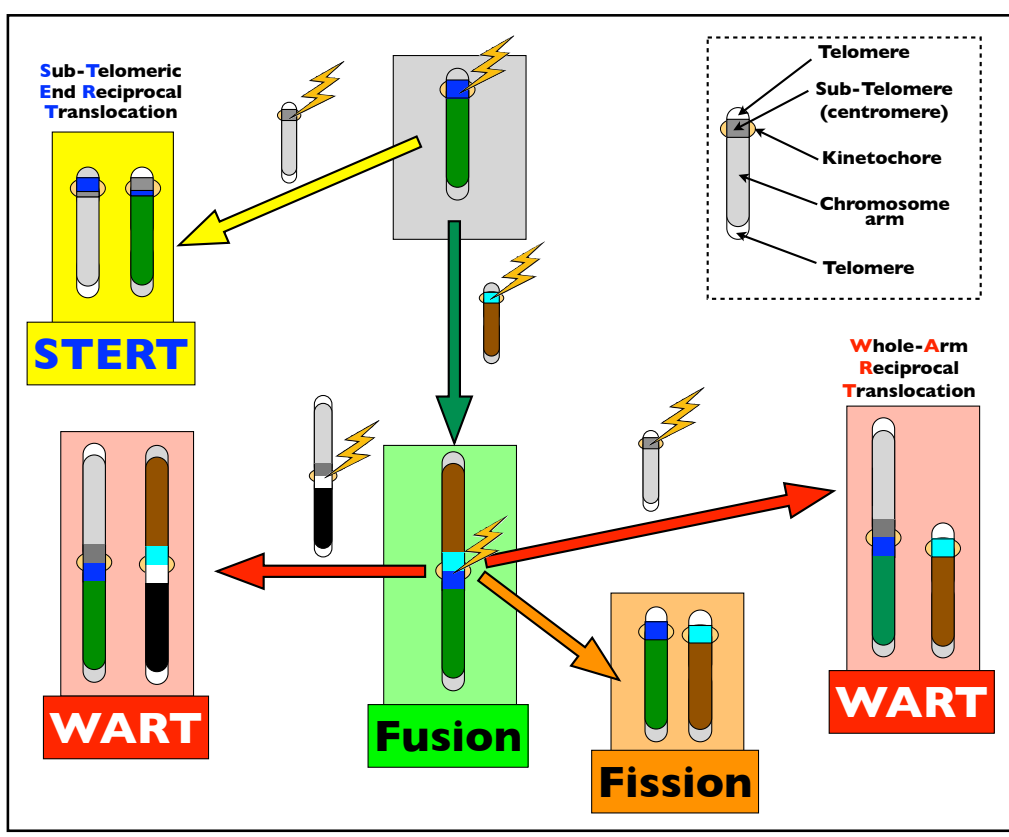

Figure 8. Double strand breaks (DSBs) in the centromeric repeat region can change chromosome structure. Simultaneous DSBS (depicted as lightning bolts) in a pair of non-homologous telocentrics can lead to reciprocal translocations that exchange the sub-telomeric ends of the chromosomes (Top-left; STERT = Sub-Telomeric End Reciprocal Translocation) or the creation of two-armed metacentric (Bottom-middle; Fusion). Simultaneous DSBs in a pair of nonhomologous metacentrics can generate an exchange of arms (Bottom-left; WART = Whole Arm Reciprocal Translocation; WARTs) and this same process between a metacentric and a telocentric moves a metacentric arm to a telocentric and the telocentric arm to the metacentric (Bottom, far-right; WART). Lastly, a metacentric can split into a pair of telocentrics (Bottom, near-right; Fission) but note that this step requires de novo telomere formation.

structure (Figure 2) will have important ramifications in the model of coevolution between centromere size and karyotype number.

Reciprocal translocations of non-homologous chromosome ends have the potential to homogenize the centromeric region among $X$ and autosomes of M. m. domesticus (Tyler-Smith and Willard 1993). I will refer to these translocations as Sub-Telomeric End Reciprocal Translocations (STERTs; Figure 8). Kalitsis et al. 2006 found extremely low sequence variation among tL1 copies found on different chromosomes (sequence divergence ranged from $0.000 \%$ to $0.002 \%)$. In addition, the sub-telocentric region as a whole was found to have the same polarity and high sequence identity (>99\%) among nonhomologous chromosomes (Kalitsis et al. 2006). The authors conclude that "This organization reflects a mechanism of frequent recombinational exchange between nonhomologous chromosomes". This high sequence similarity of chromosomal ends feasibly occurs due to swapping of subtelomeric regions between nonhomologous chromosomes, as proposed by Tyler-Smith and Willard (1993). Swapping of chromosomal ends (STERTs) would be expected to be selectively neutral (and therefore the ends would be subject to random genetic drift across different chromosomes) because the $X$ and all the autosomes share the same subtelomeric structure (containing no known genes; see Figure 2), including the same centromeric sequence (minor satellite).

Although I found no direct evidence for STERTs in the Western European house mouse, there is evidence for this process between human chromosomes. In humans, chromosomes $4 \mathrm{q}$ and $10 \mathrm{q}$ share highly similar sub-telomeric sequences. A substantial rate of sub-telomeric swaps (exchange of the entire ends of the chromosomes) between these chromosomes is demonstrated by high levels of polymorphism of swapped ends as well as individuals who are mosaic for the swap (van der Maare et al. 2000; Mefford and Trask 2002). In the model I later develop for coevolution between centromere size and karyotype number, swapping of sub-telomeric regions will have an important role in: i) reducing centromere size variation among chromosomes, and ii) returning the tL1TLC sequence to chromosomes that have lost it.

\section{Fusions and fissions of mouse chromosomes}

Both chromosomal fusions and fissions are integral parts of the model for coevolution between centromere size and karyotype number. Here I briefly review the relevant background information on these processes.

Although most Western European house mouse populations have $2 \mathrm{~N}=40$ telocentric chromosomes ( $\mathrm{X}$ and autosomes), a substantial number have $2 n<40$ with varying numbers of 
metacentrics (Pialek et al. 2005). The presence of numerous populations with at least some metacentrics indicates that fusions between telocentrics to form metacentrics occurs at nontrivial frequency in natural populations. New fusions have been observed segregating many times within laboratory strains that already contain some metacentric chromosomes $(2 \mathrm{~N}<40)$ but similar sampling effort found none in two other laboratory population that lacked metacentrics (2N = 40; Nachman and Searle 1995). Molecular analysis of naturally occurring fusions shows that their junctions consistently occur within the minor satellite region of both fused telocentrics and these fused regions do not contain any telomeric repeats nor sequences intervening between the telomere and centromere (reviewed in Garagna et al. 2014). These observations indicate that fusions between non-homologous telocentrics can and do occur in populations of M. m. domesticus, but some feature present in populations already containing at least one metacentric somehow promotes this process and/or selectively favors the retention of new metacentrics.

Fissions of metacentrics to form a pair of telocentrics has been argued to be unlikely in $M$. $m$. domesticus because they have "lost all the proximal telomeric sequences and most of the minor satellite sequences, making it unlikely that they could revert to telocentric chromosomes" (Garagna et al. 1995). However, addition of new telomere sequence to truncated mouse chromosomes is well documented (e.g., Sprung et al. 1999) and can occur at both subtelomeric and internal break points (Gao et al. 2008; Kulkarni et al. 2010). The fact that the reported size of the centromeric arrays located on mouse metacentrics are typically quite small (e.g., estimated to be $40-64 \mathrm{~kb}$ in a study by Garagna et al. 1995) would cause any new arrays located on the products of fission (new telocentrics) to be extremely small (averaging 20-32 kb). Studies of humans concerning the minimal sizes for centromeric repeats indicate that very small size ( $<50 \mathrm{~kb}$ ) would lead to sub-optimal centromeres with elevated mis-segregation rates (Lo et al. 1999; Yang et al. 2000; Okamoto et al. 2007). However, the array size of metacentric chromosomes need not be static and would feasibly be expected to expand over time (when expansion via BIR repair after fork-collapse exceed contraction due to SSA repair of DSBs) and eventually make the fission of a metacentric into a pair of telocentrics a viable outcome. This expansion will be described more fully later, when describing the coevolution model. In sum: i) the fact that the products of fission of metacentrics (segregating pairs of new telocentrics) have not been observed in natural or laboratory populations is not surprising because these metacentrics have very small centromeres which -if split into two parts- would likely produce subfunctional centromeres, but ii) fission of metacentrics to form de novo telocentrics would nonetheless be plausible after metacentrics evolved larger centromeres because studies of telomere healing demonstrate a molecular pathway to recruit de novo telomeres.

\section{Tubulin depolymerases at the kinetochore}

Movement of kinetochores as they interact with the spindle plays a central role in centromere drive of the Western European house mouse because it influences the off-center positioning of bivalents prior to anaphase-I of meiosis (Box-1, Figures 5 and 6). Tubulin depolymerases acting on microtubules generate poleward movement of kinetochores (Gorbsky et al. 1987). During mitosis, there are two tublin depolymerases that function at the kinetochore during prometaphase and metaphase that generate poleward force via the 'Pacman' depolymerization process: the kinesins Kif2b and MCAK (aka Kif2c), respectively (reviewed in Cross and McAnish 2014). Kif2b is the predominant depolymerase causing continuous tubulin turnover during prometaphase and MCAK has this role during metaphase (Bakhoum et al. 2009). A model for the switch between depolymerization via Kif2b and MCAK has been developed by Cross and McAnish 2014 and is summarized in Supplemental Figure S6.

The roles of Kif2B and MCAK in female meiosis are not well characterized (Camlin et al. 2017). However, work by Akera et al. (2019) with M. m. domesticus demonstrates that unequal recruitment of MCAK to homologs generates bivalents that are displaced off the spindle equator and toward the pole facing the homolog with the more MCAK. This data indicates that low recruitment of MCAK by the smaller kinetochores (that are recruited by very small centromeres) will cause them to lose in centromere drive (segregate to the polar body more than $50 \%$ of the time; Box-1 and Figures 5 and 6). 


\section{De novo metacentrics are predicted to sometimes win and sometimes lose in centromere drive against a pair of telocentric chromosomes}

In the late-acting form of centromere drive (Box-1), chromosomes with very small centromeres: i) recruit small kinetochores that recruit less tubulin depolymerase (MCAK), ii) produce bivalents that are displaced off the spindle equator when paired with homologs with normal-sized centromeres, and iii) ultimately lose, on average, in centromere drive -as described in Box-1 and Figures 5 and 6. Displacement of a bivalent off the spindle equator is a key feature in this form of centromere drive and also a key feature in the model for coevolution between karyotype number and centromere size. Below I explore the consequence of asymmetric KCAK recruitment on two types of metacentric chromosomes.

When a fusion between two telocentric chromosomes creates a de novo metacentric (Figure 8), its competitive ability in the context of centromere drive is expected to depend on the relative sizes of the centromeres of the three chromosomes. If all three are sufficiently large to assemble a normal-sized kinetochore, then the combined MCAK recruitment of the two telocentrics would be expected to displace the trivalent off the spindle equator toward the spindle pole closest to the pair of telocentrics (Supplemental Figure S1). For this reason, the de novo metacentric is predicted to lose in centromere drive and eventually be lost from the population.

When the telocentrics have very small centromeres -such that they recruit substantially smaller kinetochores and much less tubulin depolymerase (MCAK)- then a de novo metacentric that captures most of the centromere of each telocentric during the fusion process (Figure 8 and Supplemental Figure S2) may contain a sufficiently large centromere to recruit a full-sized kinetochore. In this case, a full-sized kinetochore at the metacentric would plausibly be able to recruit sufficiently more MCAK (compared to the combined recruitment of the two telocentrics) to displace the trivalent away from the spindle equator and toward the spindle pole facing the metacentric sister chromatids
(Supplemental Figure S2). In this context the metacentric is expected to win in centromere drive -as was observed by Harris et al. (1986) when metacentrics and telocentrics from the same population competed in centromere drive.

\section{A selection model for high local diversity with low sequence divergence among monomers}

Close proximity of centromere and telomere is expected to strongly influence repair of centromeric DSBs because they will be influenced by the TARR (Figures 1-4; see also contributing factor 2 above). TARR causes an increased incidence of resection after DSBs and also longer resection tracts (Muraki and Murnane 2017). Both of these features are expected to increase repair of DSBs via the SSA pathway. Centromeric monomers with a flanking (or close-by) identical sequence are susceptible to deletion during SSA repair of a nearby DSB (Ozenberger et al. 1991; Muchova et al. 2015; Bhargava et al. 2016). This liability generates selection (via molecular drive; Dover 1982) for sequence divergence (homeology) among monomers that are close enough to be located on opposite sides of the pair of resected regions surrounding a DSB. Because only minor sequence divergence is needed to strongly suppress homology recognition and deletion during SSA repair (Bhattacharjee et al. 2014; Bhargava et al 2016), nearby monomers do not require high dissimilarity to be protected from deletion.

Two factors can feasibly contribute to sequence divergence between a monomer and its nearby copy when a small centromeric region is tandemly duplicated. First, if the duplication occurs via BIR repair of a collapsed replication fork, this process would directly contribute to minor sequence variation among tandem duplicates because it has a base pair error rate that is $\sim 1000$-fold higher than replication without fork-collapse (Sakofsky et al. 2012). Second, and more generally, when tandemly duplicated monomers initially lack sequence divergence, then only when at least one of the monomers subsequently mutates would the duplication be expected to persist over time because only non-matching pairs would escape eventual deletion via SSArepair of nearby DSBs. Because even single base pair difference between monomers (at any of 120 positions) would be expected to strongly 
suppresses deletion via SSA-repair of DSBs, and because new mutations must occur locally within a pair of duplicated monomers, many different (high richness) low-frequency (high evenness) mutations would be expected to accumulate across the thousands of monomers located on the centromeres of the $\mathrm{X}$ and autosomes.

Recurrent deletion pressure from repair of DSBs via SSA is expected to favor multi-monomer 'neighborhoods' of non-identical sequences (Supplementary Figure S7). These would be favored by molecular drive when identical monomers are sufficiently separated (by nonmatching monomers) to prevent them from being present on opposite sides of the pair of resected regions surrounding a DSB. Selection for deletion-avoidance within tandem repeat arrays would be expected to generate the pattern of monomer diversity seen at the centromeres of $M$. $m$. domesticus: i) side-by-side monomers rarely match in sequence, ii) local pairwise sequence divergence is nearly as high as that seen globally, iii) there are many hundreds of different monomer sequences (high richness) -none of which is common (high evenness), and iv) non-identical monomers have only moderate pair-wise sequence divergence.

\section{A model for coevolution between centromere size and karyotype number}

To begin the description of the coevolutionary process, consider a population with $2 \mathrm{~N}=40$ telocentric chromosomes ( $\mathrm{X}$ and autosomes), as is currently seen in most laboratory and wild populations of $M$. m. domesticus (grey shaded region, bottom-right in Figure 9). The centromeres on all chromosomes are sufficient in length to recruit a full-sized, fully-functional kinetochore and maintain low mis-segregation rates (see Contributing Factor 7). For reasons that have not been empirically resolved, a small minority of populations experience a change that leads to the evolution of much smaller centromeres (the Contraction Trigger Event; yellow box in Figure 9). Here I hypothesize that the causative factor is something that changes the balance between BIR-expansion and SSA-contraction of the centromere (see Contributing Factor 3). Such a triggering event would plausibly represent a change that amplifies the influences of TARR on the centromere and thereby increases (to a value
$>1$ ) the ratio of centromere contraction via SSA repair of DSBs to expansions via out-of-register BIR after replication fork collapse (pink region at the bottom of Figure 9).

As an example of a plausible Contraction Triggering Event, consider a major deletion within the chromatin separating the telomere and the centromere (Figures 10D). Such a deletion could plausibly spread by drift to other telocentric autosomal chromosomes and the $X$ (but not the acrocentric $Y$ ) via STERTs (see Contributing Factor 8 and Figure 8). The deletion event would be expected to influence the contraction rate (via recurrent deletions) at the linked centromere as described in the following paragraph (see also Contributing Factor 3).

Because of the sub-telomeric location of the centromere, it is expected to be influenced by TARR due to its elevated exposure to shelterin complex proteins (especially TRF2) over at least part of its length, i.e., over its telomere-proximate region (see contributing factors 2,3 , and 5 above, and also Figure 4 and Figure 10A). In this TARRinfluenced region of the centromere, there would be increased levels of repair of DSBs via the SSA pathway (see Contributing Factor 2 and Figures 1 and 3). If this increased influence of TARR on the centromere is sufficient, it will act as a trigger that causes the centromere to begin to slowly decline in size because the rate of contraction (due to SSA repair of DSBs) is faster than than the rate of expansion (due to out-of-register BIR after the collapse of replication forks; see Contributing Factor 3). Eventually the centromeres are expected to erode to very small size and thereby recruit substantially smaller kinetochores (see Contributing Factor 7).

Very small centromeres that recruit small kinetochores are susceptible to competitive replacement via centromere drive (late-acting centromere drive; see Box-1) when segregating with chromosomes that have a substantially increased centromere and kinetochore size (see Contributing Factor 6 and Figures 5-6). Such an evolutionary replacement of smaller by larger centromeres would plausibly be suppressed, however, in the context of naturally segregating variation in centromere size because the deterministic strength of centromere drive is to too small between the largest and smallest segregating variants to overcome genetic drift 


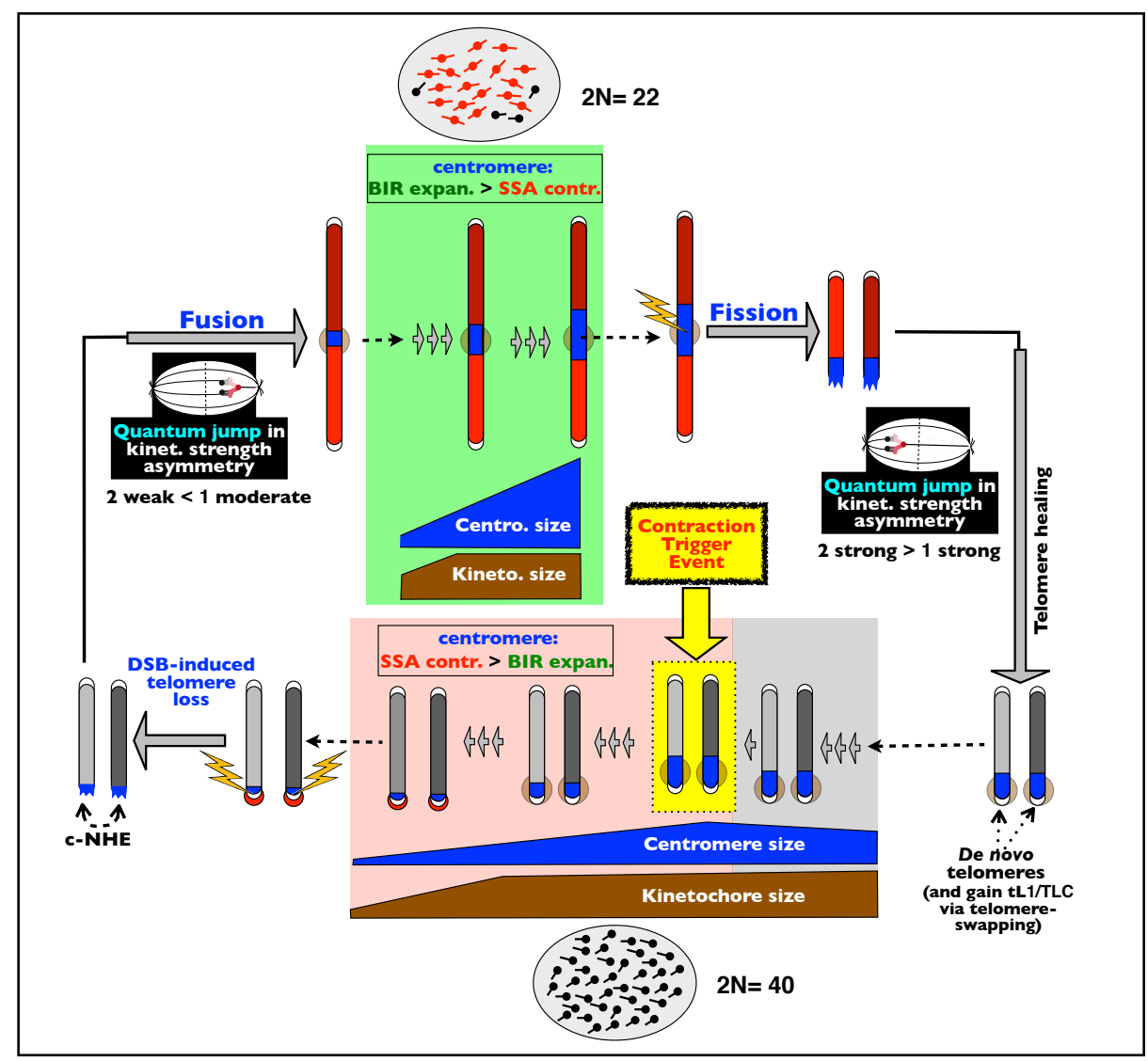

Figure 9. A model for coevolution between centromere size and karyotype number. The figure focuses on an arbitrary pair of non-homologous chromosomal arms within the genome, but the same logic applies to all autosomal arms with similar sized centromeres. The model begins with an all-telocentrics karyotype of $2 \mathrm{~N}=40$, as is typically found in most laboratory and wild populations of $M . m$. domesticus (grey shaded area, bottom-right in the figure). At this time point: i) the model assumes that STERTs have substantially homogenized the sub-telomeric regions of all 40 telocentric chromosomes so they are experiencing concerted evolution, and ii) the size of centromeric repeat arrays (centromeres) on all chromosomes are more than sufficient to recruit a full-sized/normal-functioning kinetochore (brown circles near the bottom of chromosomes). Next a "contraction trigger event" occurs (see Figure 10) that causes centromeric expansion (via out-of-register BIR after replication fork-collapse) to occur less frequently than contraction (via SSA repair of DSBs) (pink area, bottom-middle of figure). If this triggering event includes a change in sub-telomeric structure, it is assumed to have spread to all chromosomes via the STERT process. As a consequence of the contraction trigger event, the centromeres at all chromosomes begin to gradually shrink. Eventually the centromeres become so small that they assemble smaller kinetochores (depicted by the brown circles at the base of telocentrics changing to red circles) that recruit substantially reduced MCAK tubulin depolymerase. Next, DSBs within the centromeres of two nonhomologous telocentrics (both near the telomere-facing side of their minor satellite arrays) leads to their fusion, thereby creating a metacentric with a substantially larger centromere that recruits a significantly larger kinetochore (left side of Supplemental Figure S3). This 'quantum jump' in kinetochore size produces a centromere drive advantage because the de novo metacentric's kinetochore recruits sufficiently more tubulin depolymerase (MCAK) compared to the combined recruitment of the two non-fused telocentrics with small kinetochores (see Supplemental Figure S2). The asymmetry in kinetochore sizes moves the newly formed trivalent (the pair of unfused telocentrics and the newly fused metacentric; see Supplemental Figure 2) off the equator of the spindle and toward the pole facing the metacentric (MCAK recruitment inequality: 2 weak $<1$ moderate; top-left of figure). Because of its centromere drive advantage, the metacentric evolutionarily replaces the progenitor pair of telocentrics that had very small centromeres. The new metacentric configuration removes the centromere from the influence of TARR (TelomereAffected Repair Region), which reduces contraction of the centromere by SSA repair of DSBs, and allows the centromere to gradually expand in size (green box, top-middle). Kinetochore size initially increases with increasing centromere size but eventually asymptotes once a sufficient-sized centromere evolves (see contributing factor 7 above). Once the metacentric's centromere has expanded to sufficiently large size (which may take hundreds or thousands of years), the metacentric becomes susceptible to replacement via centromere drive when a fission event (in response to a DSB near the middle of the centromere) generates a pair of telocentrics that each produce a sufficiently large kinetochore -so that their combined MCAK recruitment at metaphase moves the bivalent off the middle of the spindle and toward the pole facing the pair of telocentrics (right half of figure; MCAK recruitment inequality: 2 strong $>1$ strong; top-right of figure) (also see Supplemental Figure S1). Fission requires that the new telocentrics acquire a de novo telomere (bottom-right of figure). Functional sub-telomeric features like a tL1 transposon abutting the telomere array could next be acquired by a STERT event (see Supplemental Figure S3, right half). 


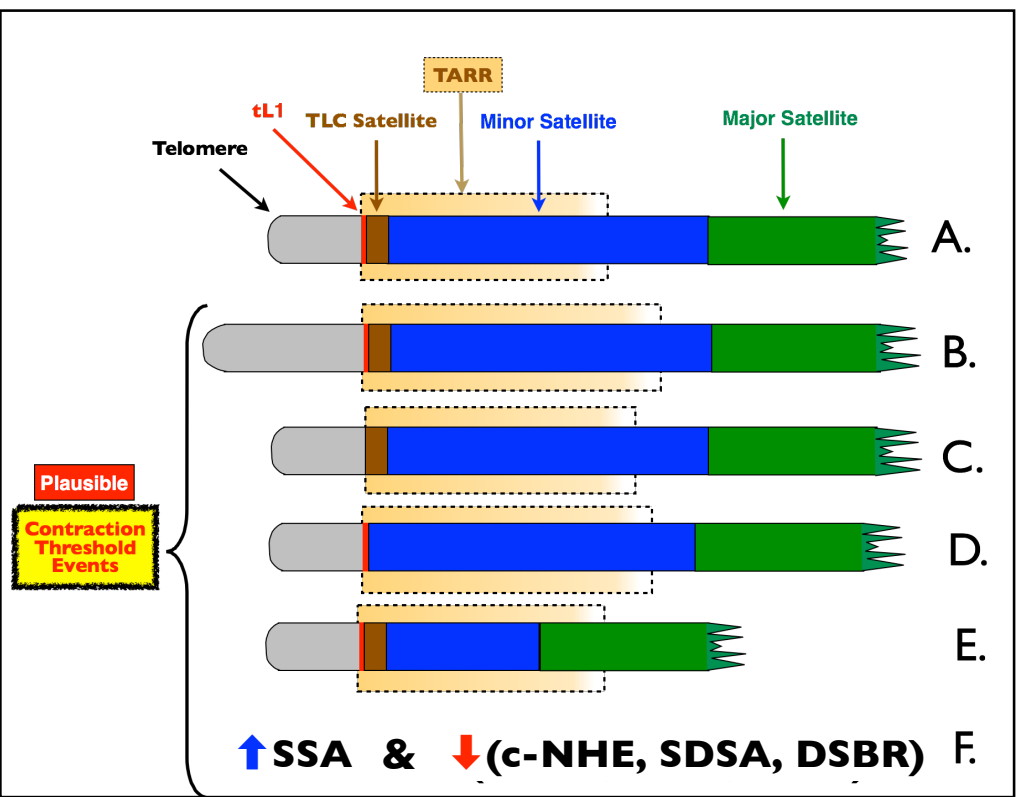

Figure 10. Plausible "Contraction threshold events" that trigger centromere erosion on telocentric chromosomes. A. Reference wild type chromosome without a contracting centromere. B. A mutation increases telomere length which increases the pool size of shelterin complex proteins and thereby expands the range of TARR. This increases the proportion of the centromere with elevated deletion rates due to SSA-repair of DSBs. C-D. A deletion removes all or part of the region separating the centromere and the telomere and thereby increases the range of TARR within the centromere. E. A major deletion reduces the size of the centromere and thereby increases the proportional range of TARR within the centromere. F. A mutation (e.g., increased production of rad52) increases the proportion of DSBs that are repaired via the SSA repair pathway rather than the c-NHEJ, Synthesis-Dependent Strand Annealing (SDSA), or Double strand Break Repair (DSBR) pathways. For B-E it is assumed that STERTs homogenize the sub-telomeric regions of all 40 telocentric chromosomes so that they evolve in concert.

(i.e., the strength of meiotic drive [when expressed as a selection coefficient] between these extremes is $<1 /\left(2 \mathrm{~N}_{\mathrm{e}}\right)$; see chapter 8 in Crow and Kimura 1970). Dallas et al. 1998 estimated the effective size of populations of $M$. $m$. domesticus with and without metacentric chromosomes to be exceptionally low $\left(N_{e}<25\right)$.

A quantum jump in centromere and kinetochore size (and hence a large change in the strength of centromere drive) would be expected to overcome the constraint of limited variation in centromere size within low- $\mathrm{N}_{\mathrm{e}}$ wild populations. Such a large-scale change is possible via fusion of two telocentrics with small centromeres to form a metacentric (Figure 8 and left side of Figure 9) with a centromere size that can potentially be as large as nearly double that of either telocentric chromosome (see Supplemental Figure S3 and Contributing Factor 9). As described above (contributing factor 9), de novo metacentrics are well documented within laboratory populations (e.g., Pialek et al. 2005; Garagna et al. 2014) and molecular work by Chmatal et al. (2014) found that in a population containing both metacentric and telocentric chromosomes, the metacentrics recruited more CENP-A and produced larger kinetochores.

Once formed, a metacentric with a substantially larger centromere and kinetochore would be expected to have the capacity to win in centromere drive assuming that the single kinetochore of the metacentric can displace the trivalent off the spindle equator toward the pole closest to the metacentric (top left in Figure 9; see also Contributing Factors 6 and 11 and Supplemental Figure S2). As described above (Contributing Factor $11)$, this result is empirically supported when metacentric and telocentrics are derived from the same wild population and placed in competition in the context of centromere drive (e.g., Harris et al. 1986). An advantage in centromere drive by a newly formed metacentric with a substantially larger centromere and kinetochore would lead to the pair of telocentrics with smaller centromeres/ kinetochores to be evolutionarily replaced by their fusion-descendent metacentric (Supplemental Figure S2).

A newly formed metacentric would lose the influence of TARR and would be expected to experience substantially less erosion via SSA repair of DSBs. As a result, expansion via out-ofregister BIR repair of collapsed replication forks could feasibly outpace contraction via SSA repair of DSBs and the centromere would gradually expand in size over time (green box in Figure 9; see also Contributing Factor 3 ). Work on the rDNA repeats of yeast indicates that this repeat array's expansion via fork-collapse/out-of-registerBIR can and does occur (Kobayashi et al. 1998) and this process is feasible at centromeres (see discussion in Rice 2019B). 
Assuming that centromeres expand while located within metacentrics, then as described above (see Contributing Factor 3), studies of human centromeres and kinetochores indicate that the kinetochores are expected to expand only to an asymptotic size despite the centromeres continued expansion (green box in figure 9; see also contributing Factor 7). Once the centromere is sufficiently large (at least double the size needed to support a normal-sized kinetochore) it becomes susceptible to invasion by pairs of de novo telocentrics (each containing a substantial portion of the metacentric's centromere) generated by fission of the metacentric (immediately followed by telomere healing; right side of Figure 9, see also Contributing Factor 9 and Figure 8). If the pair of de novo telocentrics each have sufficiently large centromeres -so that they recruit normal-sized kinetochores, they would plausibly have a centromere drive advantage because the two normal-sized kinetochores would collectively recruit double the MCAK (tubulin depolymerase) and displace the trivalent away from the spindle equator and toward the pole facing the telocentrics (twoagainst-one; Supplemental Figure S1). Once the new pair of telocentrics evolutionarily replace the metacentric due to their centromere drive advantage, the cycle is complete, and will begin again when a "contraction trigger event (see Figure 10) causes one or more telocentrics to begin gradually losing centromere length due to elevated erosion from SSA repair of DSBs due to their newly acquired TARR influence.

The cycle described above focuses on a single pair of chromosome arms. However, the cycle may be initiated for all telocentrics simultaneously. For example, suppose again that the Contraction Trigger Event (See Figure 9-10) was a major deletion in the chromatin separating the telomere and the centromere (see Figure 10D) which drifted to fixation across all autosomes and the $X$ via STERTs (Figure 8). This genetic drift is plausible in Western European house mouse populations due to their small $\mathrm{N}_{\mathrm{e}}$ (frequently $<25$; Dallas et al. 1998). Such a drift event would initiate the cycle for all 19 autosomal arms simultaneously.

Because there are 19 autosomal arms in the genome of $M$. m. domesticus (an odd number), at least one telocentric would be excluded from forming a metacentric -and therefore its centromere size would not be expected to expand due to the loss of the TARR effect that occurs in metacentrics. However the excluded telocentric could regain a large centromere by the STERTs process (see Figure 8 and Supplemental Figure S3 and S4). Such an exchange would be expected to lead to centromere drive that favored the newly formed telocentric with an expanded centromere size (Supplemental Figure S4).

The model described above does not provide a mechanism for the $\mathrm{X}$ chromosome to regain a larger centromere once it has eroded. Although more than half of all possible combinations of arms within metacentrics have been observed in natural populations of $M . m$. domesticus, the $\mathrm{X}$ is absent in all of these: presumably due to the large block of autosomal genes that would become hemizygous in males when the $X$ fuses with an autosome (Pialek et al. 2005; Garagna et al. 2014). However, similar to the process described in the previous paragraph, the $X$ could plausibly regain a large centromere by the STERTs process (see Figure 8 and Supplemental Figure S5). The resulting $X$ with an expanded centromere would be expected to have a centromere drive advantage and replace the the $X$ chromosomes with very small centromeres (Figure 5 ).

The proposed model for cyclic coevolution between centromere size and karyotype number assumes a sufficiently isolated population. If introgression between populations recurrently introduced telocentrics with normal centromere sizes, this influx would 'short-circuit' the coevolutionary cycle shown in Figure 9 by precluding the requirements for the fission and fusion mutational steps in the model. Fission and fusion are precluded because the imported telocentrics with normal-sized centromeres (wild type) would provide a simpler alternative to the formation of: i) a de novo metacentric with expanded centromere size via fusion of telocentrics with small centromeres, and ii) de novo telocentrics with normal-sized centromeres from fission of a metacentric with an expanded centromere. Unless countered by outbreeding depression at centromere-linked loci, large centromeres from imported telocentrics would also be expected to replace (via centromere drive) those from extant telocentrics and metacentrics with very small centromeres (Chmatal et al. 2014). 


\section{Discussion}

A key feature of telocentric chromosomes that motivated the hypotheses for high monomer diversity at the local level and coevolution between centromere size and karyotype number is the influence of the TARR. This phenomenon creates a fundamental difference between the centromeres of telocentrics vs. metacentrics/ acrocentrics. The operation of TARR at centromeres of $M$. m. domesticus is supported by the observation that DNA resection (and the HDrepair it initiates) is observed throughout the cell cycle at their centromeric DNA (minor satellites) unlike the more telomere-distal pericentric heterochromatin (major satellites) and most of the rest of the genome where this type of repair is restricted to the late $S$ and $G_{2}$ phases of the cell cycle (see Figure 3 and Tsouroula et al. 2016). However, evidence from human cells indicated that TARR has a restricted range of influence (Muraki and Murnane 2017) that is estimated to be at least $100 \mathrm{~kb}$ but probably less than $\sim 400 \mathrm{~kb}$. For this reason, TARR in M. m. domesticus may have an inconsequential influence on centromeres when they are separated from the telomeres by sufficient intervening DNA, or when they are sufficiently large so that much of the centromeric array is outside the TARR (Figure 4). In these cases, telocentric chromosomes would be stable because they maintain sufficient centromere size. Nonetheless, the influence of TARR would select for the high monomer diversity at the local level (clusters of monomers with nonmatching sequence) reported in the companion paper (Rice 2020).

Despite the potential stability of telocentric chromosomes, many different factors can plausibly trigger a magnified TARR influence (and act as a 'contraction trigger event'; Figure 10). A sufficient increase in the span or intensity of TARR can -in principle- initiate chronic shrinkage of the centromere (SSA contraction > BIR expansion) and initiate the hypothesized coevolution between centromere size and karyotype number (left half of Figures 9). Two important steps in this coevolution are: i) the evolution of $2 \mathrm{~N}=40$ telocentric populations that have small centromeres -as has been documented in a Greek population by Chmatal et al. (2014), and ii) the fusion of telocentrics with very small centromeres (and correspondingly small kinetochores) to generate metacentrics with substantially larger centromeres and kinetochores -as has been observed in a natural population in Switzerland (CHPO: Chmatal et al. 2014; IwataOtsubo et al. 2017).

A third important step in the model is the gradual expansion of centromeres in fusion-generated metacentrics after they have escaped the influence of TARR (i.e., when they no longer experience an elevated level of SSA repair of DSBs). To my knowledge, this key expansion step has not been directly observed and metacentrics in natural and laboratory populations have smaller centromeres than those found in all-telocentrics populations. These observations may indicate that the centromere expansion part of the coevolution model (green box) is incorrect. But there are other plausible explanations. First, natural populations with metacentrics may be too young to have evolved normal-sized centromeres via an excess of BIR expansion over SSA contraction. Second, the model predicts that metacentrics that have evolved large centromeres will be unstable and be replaced by a pair of fission-generated telocentrics because the telocentrics have a centromere drive advantage (Supplemental Figure S1). For this reason, metacentrics with large centromeres would be expected to be shortlived transients.

A second rationale to suspect that de novo metacentric centromeres eventually expand to large size (and then undergo fission back to telocentrics) is the empirical observation that most species in the genus Mus (and their subpopulations) have $2 \mathrm{~N}=40$ telocentrics. Although currently only a small proportion of a single subspecies of one species within the genus Mus is known to have evolved exceptionally small centromeres that fuse to form metacentrics, the trigger(s) that generates this evolutionary trajectory would feasibly have occurred in the lineages of all the telocentric species over the 4 million years over which this clade has evolved. If there was not a route to recover from the metacentric state, then over time a gradual evolution to all (or nearly all) metacentrics would be expected -yet this has not happened. A counter-argument to this rationale, however, is that metacentric populations are an evolutionary dead-end that eventually lead to local extinction.

One intuitive objection to the hypothesized cycle of coevolution between centromere size and 
karyotype number is the consistent presence of the $\mathrm{tL} 1$ truncated transposon that is found immediately flanking the centromere-proximate telomeres in all species (and subspecies) of the genus Mus that have been screened (Sasaki et al. 2013). This consistency across millions of years of evolution suggests that the tL 1 has some functional advantage, at least in the context of the centromere-containing end of a telocentric chromosome. However, the tL1 element is absent in the centromeric arrays of metacentrics (Garagna et al. 2014), so if their fission was to regenerate pairs of telocentrics, this feature would seem to be necessarily lost (reviewed in Garagna et al. 2014). However, the tL1 transposon may not be lost when arms of a metacentric are transformed to telocentrics (Supplemental Figure S3A), or if lost, they could readily be re-gained via STERTs (Supplemental Figure S3B) because at least one autosome and the $X$ remain telocentric in populations with metacentric chromosomes (Pialeki et al. 2005).

The hypothesized coevolution model between centromere size and karyotype number makes a number of predictions that can be tested. One prediction is that STERTs cause the centromeres to have concerted evolution in which the centromere-proximate sub-telomeric regions of the $X$ and autosomes are substantially homogenized and evolve in parallel. The extremely low sequence divergence of the tL1 truncated transposons across all $20 \mathrm{X}$ and autosomes supports this prediction, as does the fact that the remaining parts of the sub-telomeric region of these chromosomes have the same polarity and high sequence identity (>99\%; Kalitsis et al. 2006; see Contributing Factor 8). Concerted evolution of sub-telomeric regions is also supported by the observations that all chromosomes within the CHPO line (with many metacentrics) have very small centromeres simultaneously.

A second prediction is that the length of the centromere is continually eroding (although usually counterbalanced by BIR expansion), especially at its telomere-proximate end that would be expected to experience the maximal TARR influence. Empirical support for this prediction is the finding by Kalitsis et al. (2006) that monomers located near the telomereproximate ends of centromeres have high sequence similarity to the consensus. In human alpha satellite arrays (the centromeric DNA containing higher-order repeats), monomers near the edges of the array are the most disorganized and have the lowest similarity to the consensus (Schueler et al. 2001, 2005; Miga 2019) presumably because these are the oldest repeat units and so have been mostly degraded by mutations. The fact that this pattern is absent at the telomere-proximate end of the centromeric arrays of $M$. $m$. domesticus supports the prediction that this region is continually turning over via recurrent deletions, such as those generated by SSA repair of DSBs (the sink region in Figure 4).

A third prediction of the coevolution model is that SSA repair of DSBs is the process generating chronic deletions within the centromere. A genetic footprint of this process would be a strong reduction of nearby monomers with identical sequence -because close spacing makes these monomers most susceptible to deletion via SSA repair of DSBs (Schildkraut et al. 2005). The near absence of side-by-side centromeric monomers with identical sequence in Sanger traces from the mouse genome project, as well as the observation that most of global monomer sequence diversity is found within small neighborhoods of 5 monomers within Sanger traces, support this prediction (see the companion paper, Rice 2020).

An implicit assumption of the model for coevolution between centromere size and karyotype number is that some forms of centromere drive represent an adaptive process that selects against very small, sub-functional centromeres that recruit too little tubulin depolymerase (MCAK) during meiosis -with the caveat that not all forms of centromere drive are adaptive, e.g., the early-acting centromere drive described in Box-1. However, Akera et al. (2019) found no evidence for centromere drive in hybrids between $M$. $m$. domesticus males and and $M$. spretus females despite the fact that this cross produced bivalents that were offset from the spindle equator and toward the pole facing the $M$. spretus chromosomes (recruiting kinetochores with higher MKAC). Centromere drive failed to occur because it requires an asymmetry in tyrosination of the spindle that is only established during the 3-4 hour delay between the completion of metaphase-I and onset of anaphase-I -which is absent in eggs produced by $M$. spretus females. If 
adaptive centromere drive (that selectively removes very small centromeres) is present in $M$. spretus, it must be inoperative in the foreign hybrid context and not dependent upon a delayed onset of anaphase-I.

To better understand the context of centromere drive in $M . m$. domesticus, and its relationship to centromere drive in general, it would be informative to determine if the delayed onset of anaphase-I of female meiosis that is observed in this subspecies is basal within the Mus genus clade (i.e., a synapomorphy). Alternatively, this trait may be: i) independently evolved in different Mus species, ii) restricted to the species $M$. musculus, or iii) only found within $M . m$. domesticus -possibly in response to recurrent evolution of small centromeres in this subspecies. It would also be informative to determine the structure of the sub-telomeric regions separating centromeres and telomeres on telocentrics within the Mus clade members to determine whether ultra-close proximity to the telomere is unique to $M$. $m$. domesticus. Lastly, to evaluate the hypothesis that centromere drive may frequently be an adaptive process that has evolved to remove low-functioning centromeres, it would be informative to determine the fate of experimentally produced ultra-small centromeres (that recruit small kinetochores) among different species and subspecies within the genus Mus.

Centromeric repeats (minor satellite arrays) on the $\mathrm{X}$ and autosomes of $M$. m. domesticus lack HOR structure. HORs are observed at the centromere of the acrocentric $Y$ chromosome of $M$. $m$. domesticus, as well as the more telomeredistal pericentromeres (major satellite arrays) of this species (Pertile et al. 2009). HOR structure is also observed in the centromeres of all chromosomes within the the great apes clade which lack telocentric chromosomes (Archidiacono et al. 1995). These observations suggest that there is some special attribute generated by the proximity to the telomere that interferes with the evolution of HORs. A plausible hypothesis is that this attribute is the influence of TARR on centromeres of terlocentric chromosomes. Previously, I motivated the hypothesis that HORs are favored in humans via molecular drive because they increase the BIR expansion rate of subarrays that contain them more than they increase contraction rate via deletions from SSA repair of DSBs (Rice 2019B).
In the telocentrics of $M . m$. domesticus, TARR is expected to increase the contraction rate of HORs. If this increase is sufficiently high (relative to BIR expansions), it would be expected to make HORs selectively disadvantageous in the context of molecular drive.

\section{Summary and Conclusions}

This hypothesis paper concerning monomer sequence diversity and coevolution between centromere size and karyotype number was motivated by the unexpected and unusual centromere structure reported in the companion paper: the centromeric monomers in $M . m$. domesticus rarely have side-by-side monomers with identical sequence and have exceptionally high sequence diversity (as measured by monomer richness and evenness), especially at the local neighborhood level and despite only moderate average divergence of monomers from the consensus sequence. Here I hypothesized that these attributes are due to the influence of TARR on the centromeres of telocentrics. TARR causes elevated use homology-directed repair of DSBs that is expected to increases the rate of deletion via the SSA repair pathway. This single change is expected to generate: i) continuous turnover of centromeric monomers which are duplicated by out-of-register BIR after replicationfork collapse and deleted by SSA repair of DSBs, ii) selection for increased local sequence diversity (non-zero divergence among neighbors of monomers but not necessarily high levels of divergence) to depress deletions from SSA repair of DSBs, iii) selection against HOR structure when the TARR influence increases the size of deletions during SSA repair more frequently than it increases the size of expansions during BIR repair of collapsed replication forks, and plausibly iv) leads to the cascade of events described in this paper that results in coevolution between centromere size and karyotype number (summarized in Figure 9).

\section{Acknowledgments}

I thank two undergraduates working in my laboratory (Roselyn Wu and San Ha Lee) for assistance during the exploratory stages of the sequence analysis described in the companion paper. I also thank Kathryn Schoenrock for copyediting assistance. 


\section{References}

Aker, M., \& Huang, H. V. (1996). Extreme heterogeneity of minor satellite repeat arrays in inbred strains of mice. Mammalian Genome, 7(1), 62-64.

Akera, T., Trimm, E., \& Lampson, M. A. (2019). Molecular strategies of meiotic cheating by selfish centromeres. Cell, 178(5), 1132-1144.

Akera, T., Chmátal, L., Trimm, E., Yang, K., Aonbangkhen, C., Chenoweth, D. M., ... \& Lampson, M. A. (2017). Spindle asymmetry drives non-Mendelian chromosome segregation. Science, 358(6363), 668-672.

Archidiacono N, Antonacci R, Marzella R, et al. (1995) Comparative mapping of human alphoid sequences in great apes using fluorescence in situ hybridization. Genomics 25, 477-484.

Aze, A., Sannino, V., Soffientini, P., Bachi, A., \& Costanzo, V. (2016). Centromeric DNA replication reconstitution reveals DNA loops and ATR checkpoint suppression. Nature cell biology, 18(6), 684-691.

Bakhoum, S. F., Thompson, S. L., Manning, A. L., \& Compton, D. A. (2009). Genome stability is ensured by temporal control of kinetochoremicrotubule dynamics. Nature cell biology, 11(1), 27-35.

Bhargava, R., Onyango, D. O., \& Stark, J. M. (2016). Regulation of single-strand annealing and its role in genome maintenance. Trends in Genetics, 32(9), 566-575.

Bhattacharjee, V., Lin, Y., Waldman, B. C., \& Waldman, A. S. (2014). Induction of recombination between diverged sequences in a mammalian genome by a double-strand break. Cellular and molecular life sciences, 71(12), 2359-2371.

Bodor, D. L., Mata, J. F., Sergeev, M., David, A. F., Salimian, K. J., Panchenko, T., ... \& Jansen, L. E. (2014). The quantitative architecture of centromeric chromatin. Elife, 3, e02137.

Bouck, D. C., \& Bloom, K. (2007). Pericentric chromatin is an elastic component of the mitotic spindle. Current biology, 17(9), 741-748.

Burt, A., \& Trivers, R. (2006). Genes in Conflict: The Biology of Selfish Genetic Elements (Belknap, Cambridge, MA).

Calado, R. T., \& Dumitriu, B. (2013, April). Telomere dynamics in mice and humans. In Seminars in hematology (Vol. 50, No. 2, pp. 165-174). WB Saunders.

Camlin, N. J., McLaughlin, E. A., \& Holt, J. E. (2017). Motoring through: the role of kinesin superfamily proteins in female meiosis. Human reproduction update, 23(4), 409-420.
Ceccaldi, R., Rondinelli, B., \& D'Andrea, A. D. (2016). Repair pathway choices and consequences at the double-strand break. Trends in cell biology, 26(1), 52-64.

Chmátal, L., Gabriel, S. I., Mitsainas, G. P., MartínezVargas, J., Ventura, J., Searle, J. B., ... \& Lampson, M. A. (2014). Centromere strength provides the cell biological basis for meiotic drive and karyotype evolution in mice. Current Biology, 24(19), 2295-2300.

Chmátal, L., Yang, K., Schultz, R. M., \& Lampson, M. A. (2015). Spatial regulation of kinetochore microtubule attachments by destabilization at spindle poles in meiosis I. Current Biology, 25(14), 1835-1841.

Chmátal, L., Schultz, R. M., Black, B. E., \& Lampson, M. A. (2017). Cell biology of cheatingtransmission of centromeres and other selfish elements through asymmetric meiosis. In Centromeres and Kinetochores (pp. 377-396). Springer, Cham.

Crosetto, N., Mitra, A., Silva, M. J., Bienko, M., Dojer, N., Wang, Q., ... \& Pasero, P. (2013). Nucleotideresolution DNA double-strand break mapping by next-generation sequencing. Nature methods, 10(4), 361.

Cross, R. A., \& McAinsh, A. (2014). Prime movers: the mechanochemistry of mitotic kinesins. Nature reviews Molecular cell biology, 15(4), 257-271.

Crow, J. F., \& Kimura, M. (1970). An introduction to population genetics theory. An introduction to population genetics theory.

Dallas, J. F., Bonhomme, F., Boursot, P., BrittonDavidian, J., \& Bauchau, V. (1998). Population genetic structure in a Robertsonian race of house mice: evidence from microsatellite polymorphism. Heredity, 80(1), 70-77.

de Lange, T. (2018). Shelterin-mediated telomere protection. Annual review of genetics, 52, 223-247.

Dover, G. (1982). Molecular drive: a cohesive mode of species evolution. Nature, 299(5879), 111-117.

Gao, Q., Reynolds, G. E., Wilcox, A., Miller, D., Cheung, P., Artandi, S. E., \& Murnane, J. P. (2008). Telomerase-dependent and-independent chromosome healing in mouse embryonic stem cells. DNA repair, 7(8), 1233-1249.

Garagna, S., Broccoli, D., Redi, C. A., Searle, J. B., Cooke, H. J., \& Capanna, E. (1995). Robertsonian metacentrics of the house mouse lose telomeric sequences but retain some minor satellite DNA in the pericentromeric area. Chromosoma, 103(10), 685-692.

Garagna, S., Page, J., Fernandez-Donoso, R., Zuccotti, M., \& Searle, J. B. (2014). The Robertsonian phenomenon in the house mouse: mutation, meiosis and speciation. Chromosoma, 123(6), 529-544. 
Gorbsky, G. J., Sammak, P. J., \& Borisy, G. G. (1987). Chromosomes move poleward in anaphase along stationary microtubules that coordinately disassemble from their kinetochore ends. The Journal of cell biology, 104(1), 9-18.

Greenfeder, S. A., \& Newlon, C. S. (1992). Replication forks pause at yeast centromeres. Molecular and cellular biology, 12(9), 4056-4066.

Harris, M. J., Wallace, M. E., \& Evans, E. P. (1986). Aneuploidy in the embryonic progeny of females heterozygous for the Robertsonian chromosome (9.12) in genetically wild Peru-Coppock mice (Mus musculus). Reproduction, 76(1), 193-203.

Henikoff, S., Ahmad, K., \& Malik, H. S. (2001). The centromere paradox: stable inheritance with rapidly evolving DNA. Science, 293(5532), 1098-1102.

Iwata-Otsubo, A., Dawicki-McKenna, J. M., Akera, T., Falk, S. J., Chmátal, L., Yang, K., ... \& Black, B. E. (2017). Expanded satellite repeats amplify a discrete CENP-A nucleosome assembly site on chromosomes that drive in female meiosis. Current Biology, 27(15), 2365-2373.

Jain, S., Sugawara, N., \& Haber, J. E. (2016). Role of double-strand break end-tethering during gene conversion in Saccharomyces cerevisiae. PLOS genetics, 12(4).

Kalitsis, P., Griffiths, B., \& Choo, K. A. (2006). Mouse telocentric sequences reveal a high rate of homogenization and possible role in Robertsonian translocation. Proceedings of the National Academy of Sciences, 103(23), 8786-8791.

Kent, I. A., \& Lele, T. P. (2017). Microtubule-based force generation. Wiley Interdisciplinary Reviews: Nanomedicine and Nanobiotechnology, 9(3), e1428.

Kipling, D., Ackford, H. E., Taylor, B. A., \& Cooke, H. J. (1991). Mouse minor satellite DNA genetically maps to the centromere and is physically linked to the proximal telomere. Genomics, 11(2), 235-241.

Kipling, D., Wilson, H. E., Mitchell, A. R., Taylor, B. A., \& Cooke, H. J. (1994). Mouse centromere mapping using oligonucleotide probes that detect variants of the minor satellite. Chromosoma, 103(1), 46-55.

Kobayashi, T. (2014). Ribosomal RNA gene repeats, their stability and cellular senescence. Proceedings of the Japan Academy, Series B, 90(4), 119-129.

Kobayashi, T., Heck, D. J., Nomura, M., \& Horiuchi, T. (1998). Expansion and contraction of ribosomal DNA repeats in Saccharomyces cerevisiae: requirement of replication fork blocking (Fob1) protein and the role of RNA polymerase I. Genes \& development, 12(24), 3821-3830.
Kobayashi, T., \& Ganley, A. R. (2005). Recombination regulation by transcription-induced cohesin dissociation in rDNA repeats. Science, 309(5740), 1581-1584.

Komissarov, A. S., Gavrilova, E. V., Demin, S. J., Ishov, A. M., \& Podgornaya, O. I. (2011). Tandemly repeated DNA families in the mouse genome. BMC genomics, 12(1), 531.

Kulkarni, A., Zschenker, O., Reynolds, G., Miller, D., \& Murnane, J. P. (2010). Effect of telomere proximity on telomere position effect, chromosome healing, and sensitivity to DNA double-strand breaks in a human tumor cell line. Molecular and cellular biology, 30(3), 578-589.

Kuznetsova, I., Podgornaya, O., \& Ferguson-Smith, M. A. (2006). High-resolution organization of mouse centromeric and pericentromeric DNA. Cytogenetic and genome research, 112(3-4), 248-255.

Lampson, M. A., \& Black, B. E. (2017, January). Cellular and molecular mechanisms of centromere drive. In Cold Spring Harbor symposia on quantitative biology (Vol. 82, pp. 249-257). Cold Spring Harbor Laboratory Press.

Lo, A. W., Liao, G. C. C., Rocchi, M., \& Choo, K. A. (1999). Extreme reduction of chromosomespecific a-satellite array is unusually common in human chromosome 21. Genome Research, 9(10), 895-908.

Lo, A. W., Sprung, C. N., Fouladi, B., Pedram, M., Sabatier, L., Ricoul, M., ... \& Murnane, J. P. (2002). Chromosome instability as a result of double-strand breaks near telomeres in mouse embryonic stem cells. Molecular and cellular biology, 22(13), 4836-4850.

Marciniak, M., Lenartowicz, M., Golas, A., \& Styrna, J. (2010). Correlation of centromeric heterochromatin C-band polymorphism with breeding failure in mice. Folia biologica, 58(3-4), 251-255.

Marzais, B., Vorsanova, S. G., Roizes, G., \& Yurov, Y. B. (1999). Analysis of alphoid DNA variation and kinetochore size in human chromosome 21: evidence against pathological significance of alphoid satellite DNA diminutions. TSitologiia $i$ genetika, 33(1), 25-31.

Mefford, H. C., \& Trask, B. J. (2002). The complex structure and dynamic evolution of human subtelomeres. Nature Reviews Genetics, 3(2), 91-102.

Miga, K. H., Newton, Y., Jain, M., Altemose, N., Willard, H. F., \& Kent, W. J. (2014). Centromere reference models for human chromosomes $X$ and $Y$ satellite arrays. Genome research, 24(4), 697-707.

Miga, K. H. (2019). Centromeric satellite DNAs: hidden sequence variation in the human population. Genes, 10(5), 352. 
Mitra, S., Gomez-Raja, J., Larriba, G., Dubey, D. D., \& Sanyal, K. (2014). Rad51-Rad52 mediated maintenance of centromeric chromatin in Candida albicans. PLoS genetics, 10(4).

Muchová, V., Amiard, S., Mozgová, I., Dvořáčková, M., Gallego, M. E., White, C., \& Fajkus, J. (2015). Homology-dependent repair is involved in $45 \mathrm{~S}$ rDNA loss in plant CAF-1 mutants. The Plant Journal, 81(2), 198-209.

Muraki, K., \& Murnane, J. P. (2017). The DNA damage response at dysfunctional telomeres, and at interstitial and subtelomeric DNA double-strand breaks. Genes \& genetic systems, 17-00014.

Nachman, M. W., \& Searle, J. B. (1995). Why is the house mouse karyotype so variable?. Trends in ecology \& evolution, 10(10), 397-402.

Oguchi, Y., Uchimura, S., Ohki, T., Mikhailenko, S. V., \& Ishiwata, S. I. (2011). The bidirectional depolymerizer MCAK generates force by disassembling both microtubule ends. Nature cell biology, 13(7), 846-852.

Okamoto, Y., Nakano, M., Ohzeki, J. I., Larionov, V., \& Masumoto, H. (2007). A minimal CENP-A core is required for nucleation and maintenance of a functional human centromere. The EMBO journal, 26(5), 1279-1291.

Ozenberger, B. A., \& Roeder, G. S. (1991). A unique pathway of double-strand break repair operates in tandemly repeated genes. Molecular and cellular biology, 11(3), 1222-1231.

Peris, L., Wagenbach, M., Lafanechère, L., Brocard, J., Moore, A. T., Kozielski, F., ... \& Andrieux, A. (2009). Motor-dependent microtubule disassembly driven by tubulin tyrosination. Journal of Cell Biology, 185(7), 1159-1166.

Pertile, M. D., Graham, A. N., Choo, K. A., \& Kalitsis, P. (2009). Rapid evolution of mouse $Y$ centromere repeat DNA belies recent sequence stability. Genome research, 19(12), 2202-2213.

Piálek, J. A. R. O. S. L. A. V., Hauffe, H. C., \& Searle, J. B. (2005). Chromosomal variation in the house mouse. Biological Journal of the Linnean Society, 84(3), 535-563

Rice, W. R. (2019A). A Game of Thrones at Human Centromeres I. Multifarious structure necessitates a new molecular/evolutionary model. BioRxiv, 731430

Rice, W. R. (2019B). A Game of Thrones at Human Centromeres II. A new molecular/evolutionary model. BioRxiv, 731471.

Rice (2020) Centromeric repeats of the Western European house mouse I: unusual repeat structure due to extreme sequence diversity among monomers at local and global spatial scales. BioRxiv, NNNNNN.

Rosin, L. F., \& Mellone, B. G. (2017). Centromeres drive a hard bargain. Trends in Genetics, 33(2), 101-117.
Sasaki, N., Yamauchi, H., Tomohiro, N., \& Agui, T. (2013). The telocentric tandem repeat at the $p$ arm is not conserved in Mus musculus subspecies. Gene, 513(1), 214-218.

Sakofsky CJ, Ayyar S, Malkova A (2012) Break-induced replication and genome stability. Biomolecules 2 , 483-504.

Schildkraut, E., Miller, C. A., \& Nickoloff, J. A. (2005). Gene conversion and deletion frequencies during double-strand break repair in human cells are controlled by the distance between direct repeats. Nucleic acids research, 33(5), 1574-1580.

Schueler, M. G., Higgins, A. W., Rudd, M. K., Gustashaw, K., \& Willard, H. F. (2001). Genomic and genetic definition of a functional human centromere. Science, 294(5540), 109-115.

Schueler, M. G., Dunn, J. M., Bird, C. P., Ross, M. T., Viggiano, L., Rocchi, M., ... \& NISC Comparative Sequencing Program. (2005). Progressive proximal expansion of the primate $\mathrm{X}$ chromosome centromere. Proceedings of the National Academy of Sciences, 102(30), 10563-10568.

Silva, B. A., Jones, T. J., \& Murnane, J. P. (2017). Differences in the recruitment of DNA repair proteins at subtelomeric and interstitial I-Scel endonuclease-induced DNA double-strand breaks. DNA repair, 49, 1-8.

Sirajuddin, M., Rice, L. M., \& Vale, R. D. (2014). Regulation of microtubule motors by tubulin isotypes and post-translational modifications. Nature cell biology, 16(4), 335-344

Smith, G. P. (1976). Evolution of repeated DNA sequences by unequal crossover. Science, 191(4227), 528-535.

Sprung, C. N., Reynolds, G. E., Jasin, M., \& Murnane, J. P. (1999). Chromosome healing in mouse embryonic stem cells. Proceedings of the National Academy of Sciences, 96(12), 6781-6786.

Stephens, A. D., Haggerty, R. A., Vasquez, P. A., Vicci, L., Snider, C. E., Shi, F., ... \& Verdaasdonk, J. S. (2013). Pericentric chromatin loops function as a nonlinear spring in mitotic force balance. Journal of Cell Biology, 200(6), 757-772.

Thilagavathi, J., Venkatesh, S., \& Dada, R. (2013). Telomere length in reproduction. Andrologia, 45(5), 289-304.

Tsouroula, K., Furst, A., Rogier, M., Heyer, V., MaglottRoth, A., Ferrand, A., ... \& Soutoglou, E. (2016). Temporal and spatial uncoupling of DNA double strand break repair pathways within mammalian heterochromatin. Molecular cell, 63(2), 293-305.

Tyler-Smith, C., \& Willard, H. F. (1993). Mammalian chromosome structure. Current opinion in genetics \& development, 3(3), 390-397.

van der Maarel, S. M., Deidda, G., Lemmers, R. J., van Overveld, P. G., van der Wielen, M., Hewitt, J. E., ... \& Frants, R. R. (2000). De novo 
facioscapulohumeral muscular dystrophy: frequent somatic mosaicism, sex-dependent phenotype, and the role of mitotic transchromosomal repeat interaction between chromosomes 4 and 10. The American Journal of Human Genetics, 66(1), 26-35.

Waldman, A. S., \& Liskay, R. M. (1988). Dependence of intrachromosomal recombination in mammalian cells on uninterrupted homology. Molecular and cellular biology, 8(12), 5350-5357.

Wang RW, Lee C-S, Haber JE (2017) Position effects influencing intrachromosomal repair of a doublestrand break in budding yeast. PLOS ONE 12, e0180994.

Willard, H. F. (1991). Evolution of alpha satellite. Current opinion in genetics \& development, 1(4), 509-514.

Wu, T., Lane, S. I., Morgan, S. L., \& Jones, K. T. (2018). Spindle tubulin and MTOC asymmetries may explain meiotic drive in oocytes. Nature communications, 9(1), 1-11.

Yang, J. W., Pendon, C., Yang, J., Haywood, N., Chand, A., \& Brown, W. R. A. (2000). Human mini-chromosomes with minimal centromeres. Human Molecular Genetics, 9(12), 1891-1902.

Zschenker, O., Kulkarni, A., Miller, D., Reynolds, G. E., Granger-Locatelli, M., Pottier, G., ... \& Murnane, J. P. (2009). Increased sensitivity of subtelomeric regions to DNA double-strand breaks in a human cancer cell line. DNA repair, 8(8), 886-900. 


\section{Supplementary Figures}

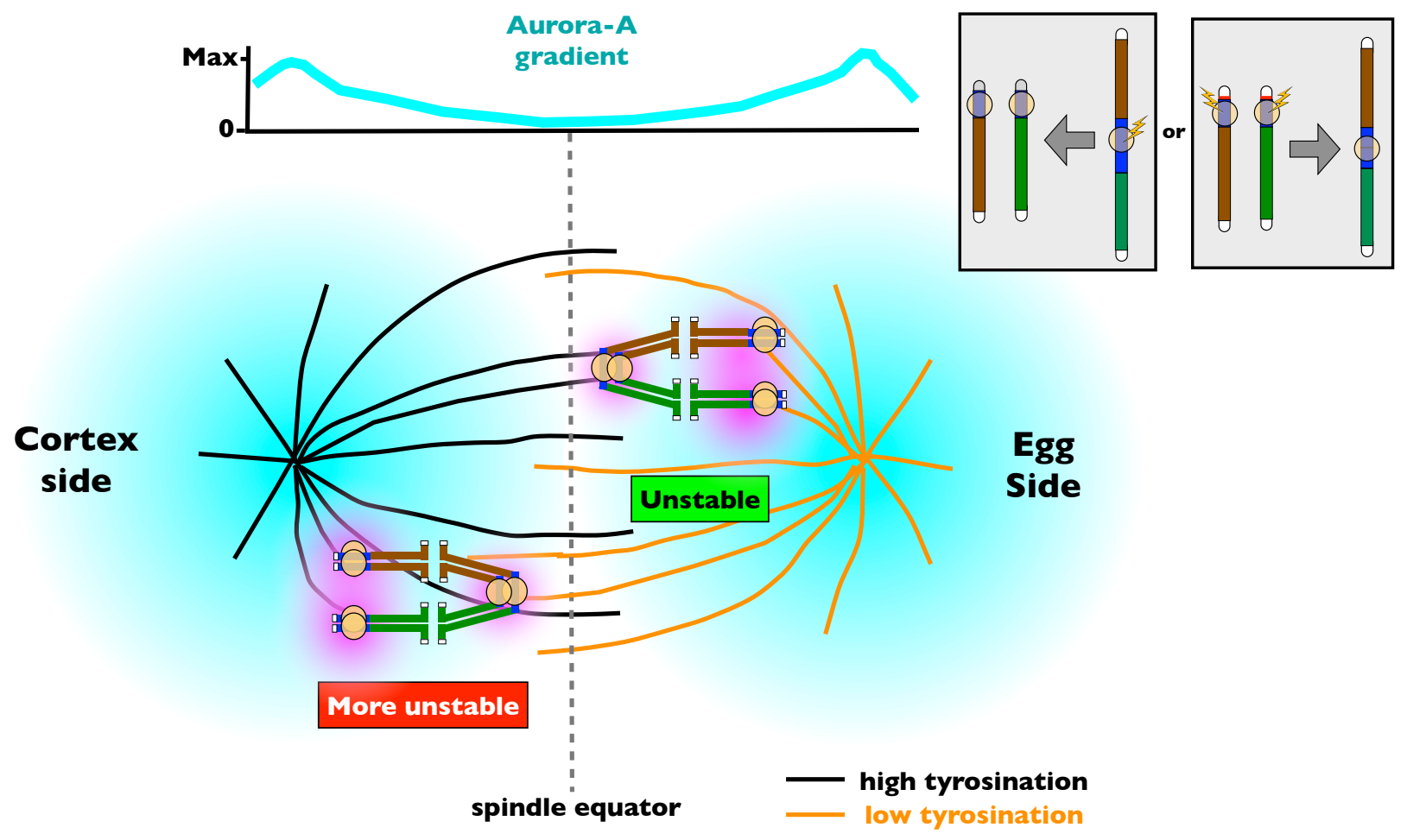

Supplemental Figure S1. When all kinetochores are the same size, a pair of telocentrics is expected to win (segregate to the egg more than $50 \%$ of the time) in late-acting centromere drive because they will have a combined stronger MCAK recruitment within trivalents (2 strong $>1$ strong). See Figure 5 for additional details and labelling. 


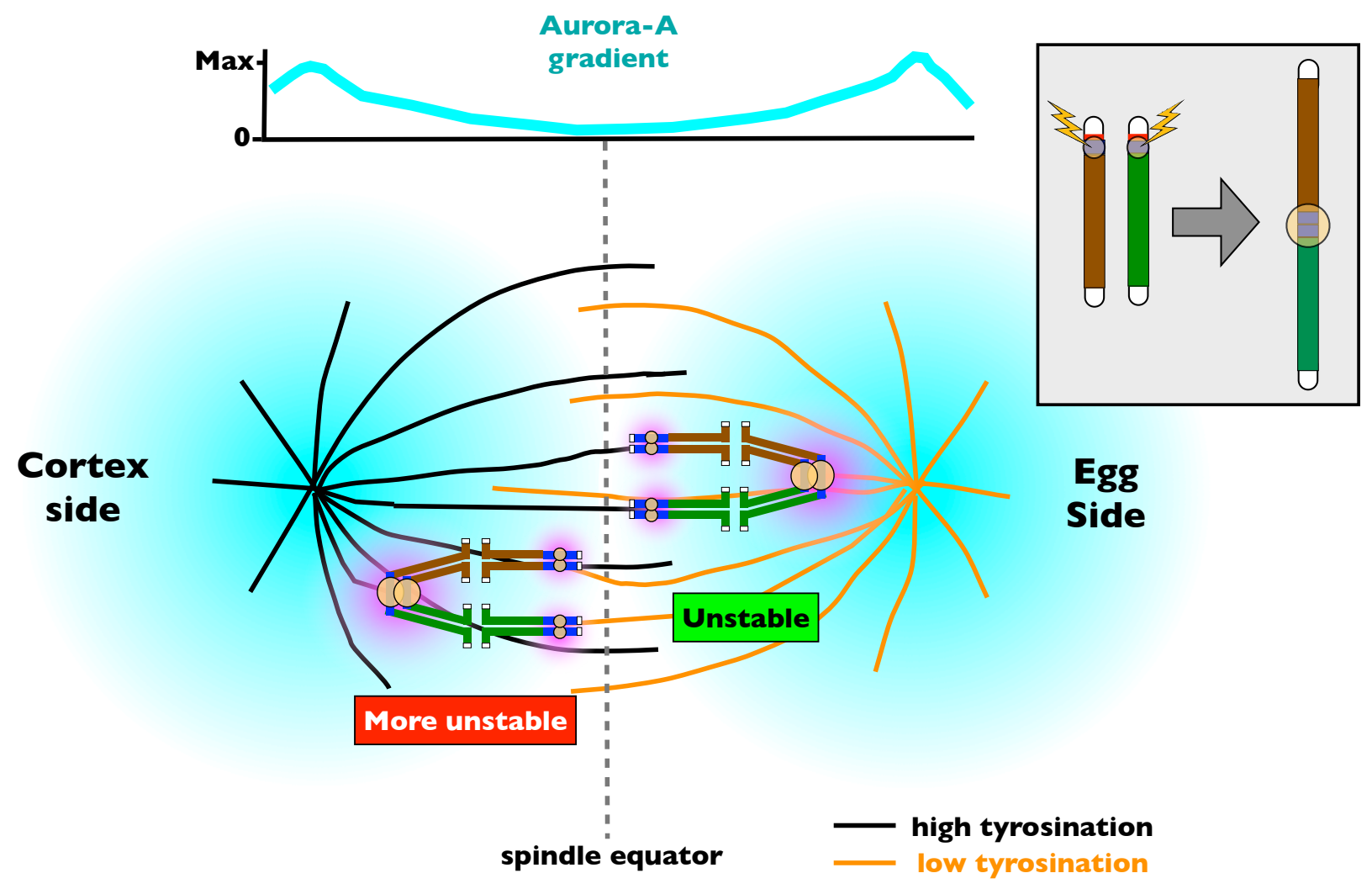

Supplemental Figure S2. When the kinetochore of a metacentric is sufficiently larger than that of both telocentrics, the metacentric is expected to win (segregate to the egg more than $50 \%$ of the time) in late-acting centromere drive because it will have stronger MCAK recruitment within trivalents ( 1 strong $>2$ weak). See Figure 5 for additional details and labelling. 


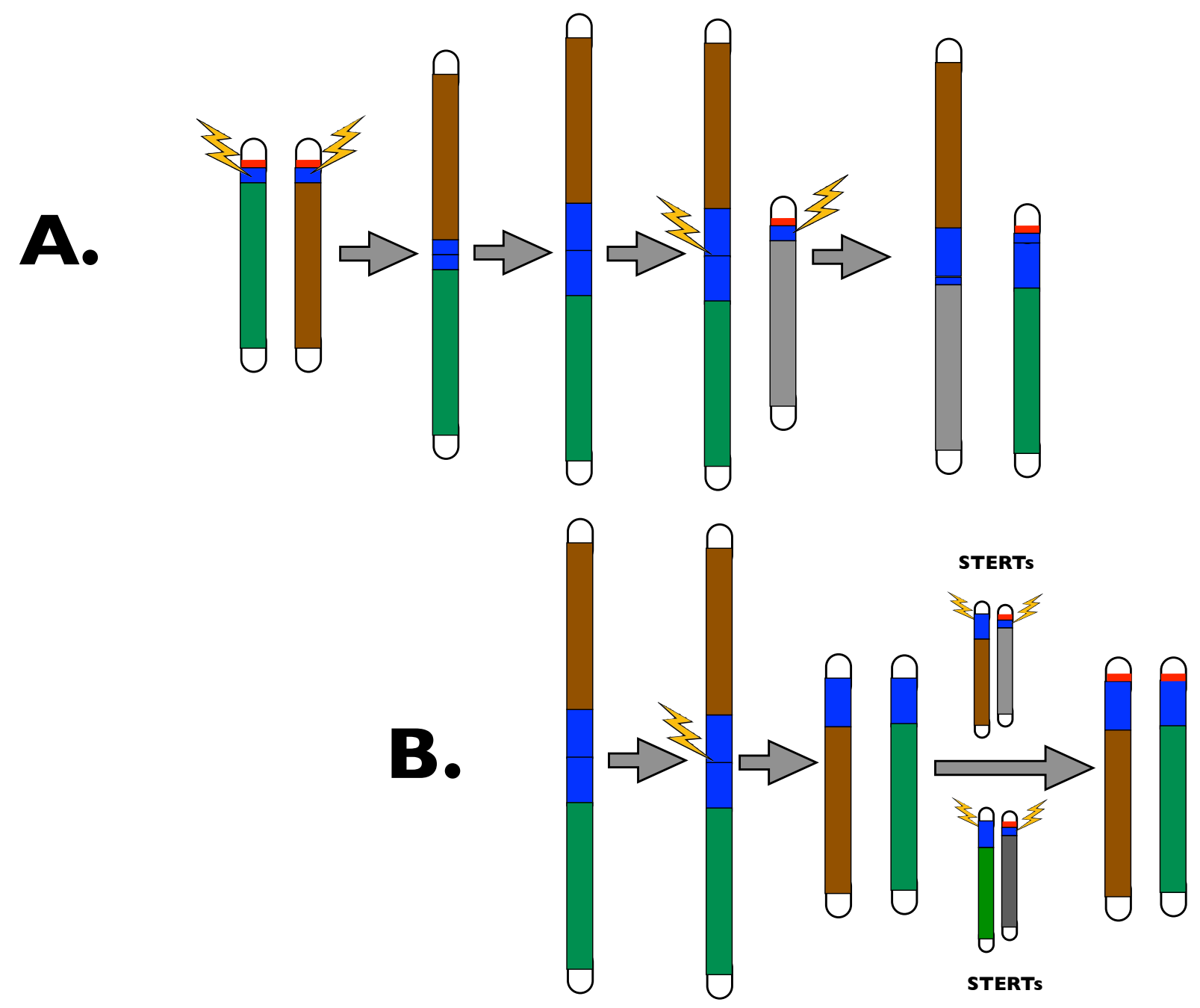

Supplemental Figure S3. A. Left: Fusion of telocentrics can produce a metacentric with a substantially larger centromere. A. Middle: The centromere of a metacentric can potentially expand because it does not experience TARR and hence has a lower contraction rate due to SSA repair of DSBs. A. Right: The WART process can exchange an arm of a metacentric with the arm of a telocentric and produce a new telocentric with an expanded centromere. B. If fusion of telocntrics generates a pair of telocentrics without the tL1 element (or a TLC repeat array), this feature can be regenerated vis STERTs. 


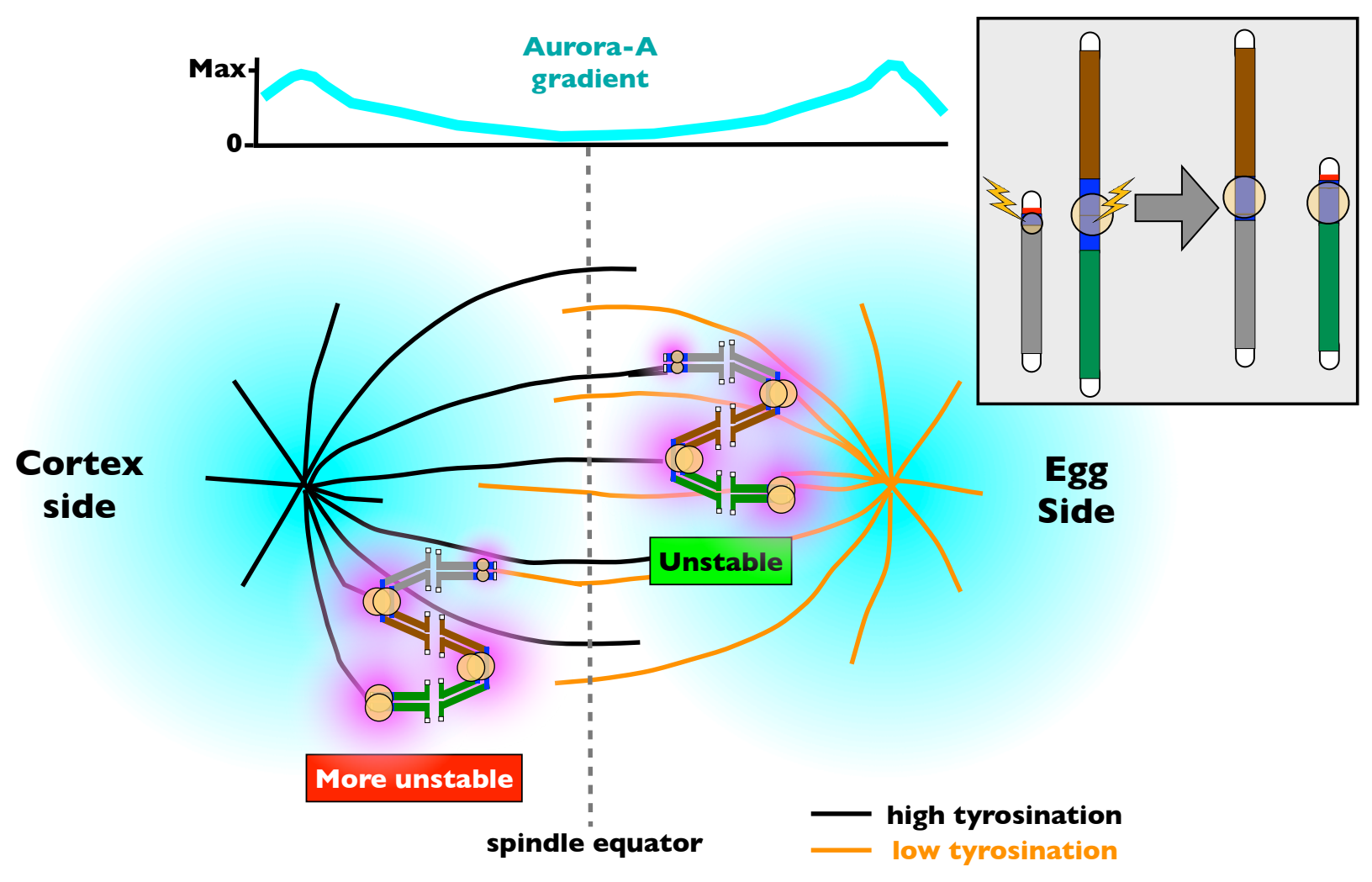

Supplemental Figure S4. When a telocentric with a very small centromere (that recruits a small kinetochore) undergoes WART with a metacentric with a substantially larger centromere, the newly formed metacentric/telocentric pair is expected to have: i) both members with sufficiently large centromeres to recruit normal-sized kinetochores (strong kinetochores), and ii) a centromere drive advantage against the unchanged pair of chromosomes due to stronger MCAK recruitment $(2$ strong $>(1$ strong +1 weak). See Figure 5 for additional details and labelling. 


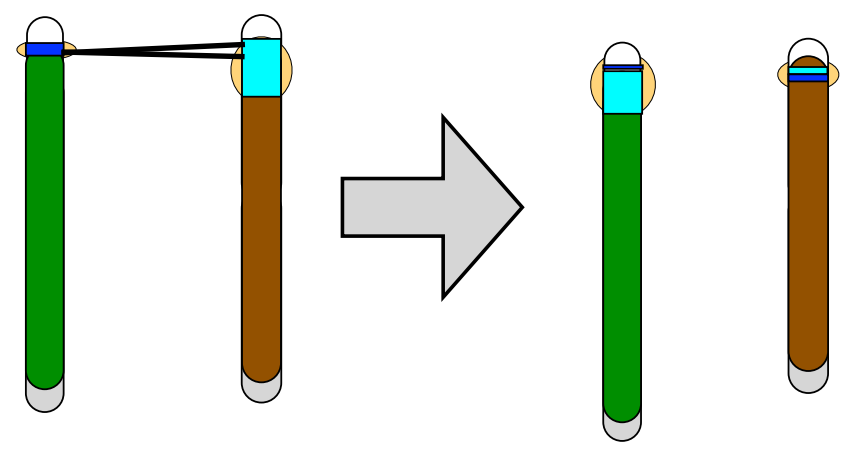

Supplemental Figure S5. STERTs between a telocentric with very small centromeres and another non-homolog with a large centromere can transfer the large centromere between the chromosomes. This process would be expected to enable chromosomes like the $X$ (when it has a very small centromere) to acquire an expanded centromere size. 


\section{A. Prometaphase}

(low kinetochore separation)

\section{B. Metaphase}

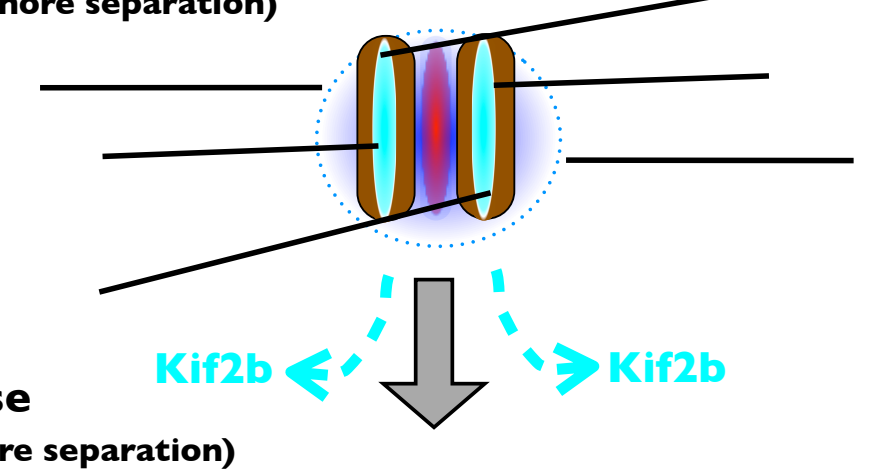

\section{(high kinetochore separation)}
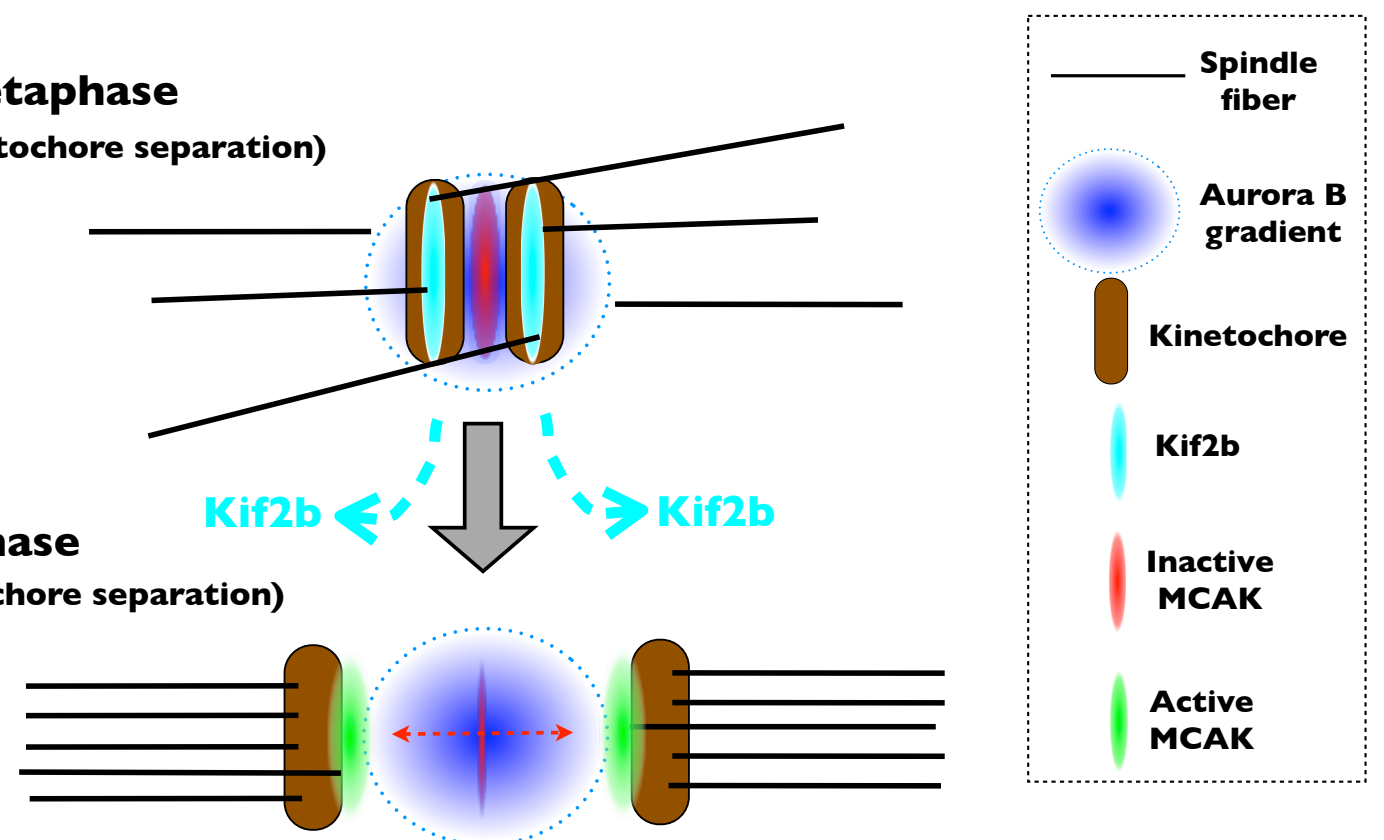

Supplemental Figure S6. A model for depolymerase recruitment at kinetochores. A. During prometaphase of mitosis, kinetochores are closely spaced and reside within a pool of Aurora-B kinase. Contact with Aurora-B deactivates MCAK and causes the the recruitment of Kif2B. So at this time point, Kif2B is the active tubulin depolymerase. B. During metaphase, bivalents are bioriented and stretched which moves the kinetochores out of contact with Aurora-B and activates the associated MCAK and depletes the Kif2B. So at this time point, MCAK is the active tubulin depolymerase. This model based on one initially proposed by Bakhoum et al. (2009). 


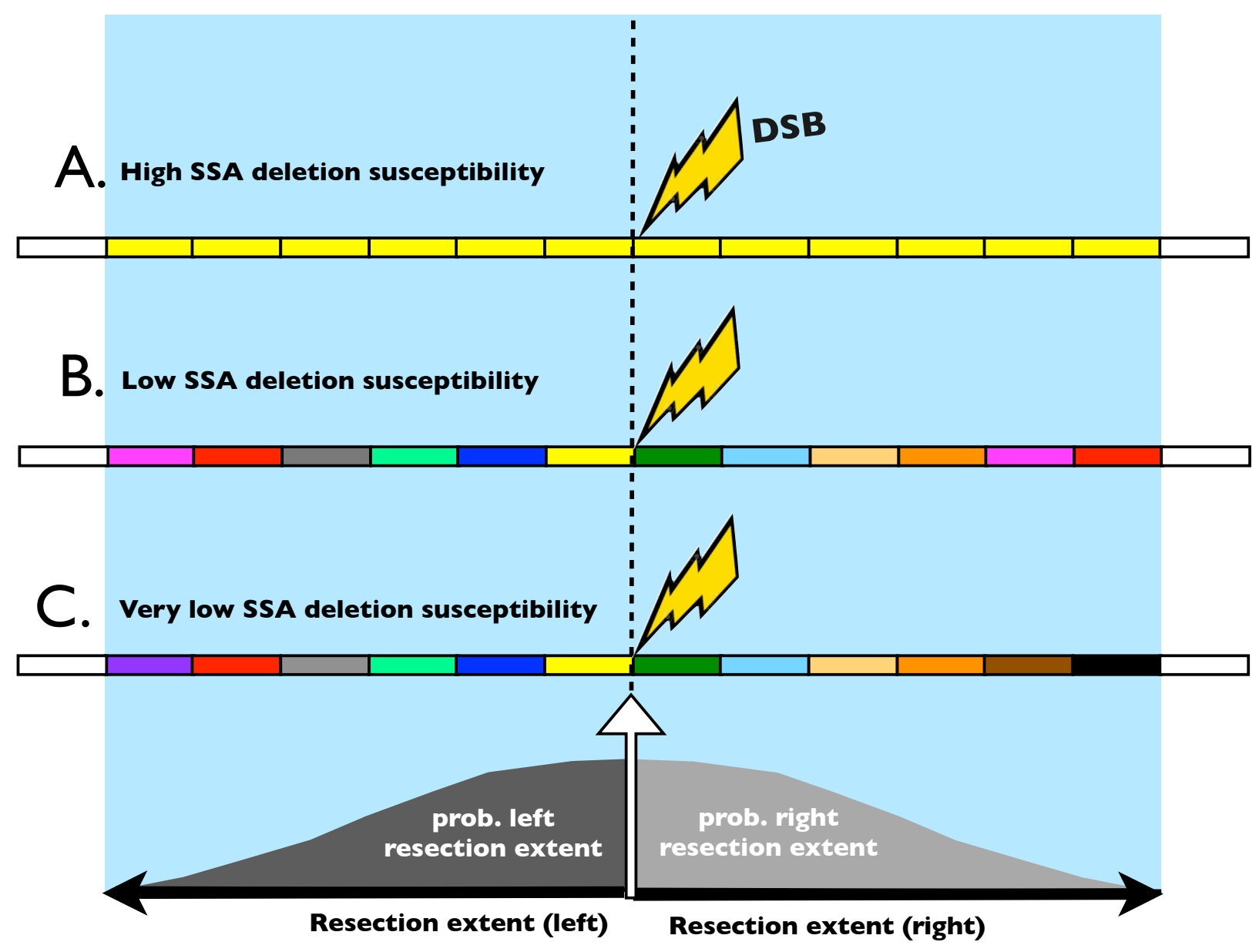

Supplemental Figure S7. Model for a molecular-drive advantage (lower deletion rate) of neighborhoods with non-matching monomers. Clusters of centromeric monomers (neighborhoods) with increased distance between matching sequences $(A<B<C)$ have increased protection from deletion via SSA-repair of DSBs. SSA-induced deletions predominantly occur when one or more monomers are matching on the left and right resected tracts surrounding a DSB. Colored rectangles depict monomers and different colored rectangles depict non-matching sequences. The graph at the bottom (probability [vertical axis] vs. distance from DSB [horizontal axis]) depicts an expected declining probability of left and right resection tracts extending to the length indicated by the position on the $\mathrm{X}$ axis (resection extent). 